\title{
Resistive vibration exercise during bed-rest reduces motor control changes in the lumbo-pelvic musculature
}

\author{
Daniel L. Belavý a,b,c,*, Stephen J. Wilson ${ }^{\mathrm{c}}$, Gabriele Armbrecht ${ }^{\mathrm{a}}$, Jörn Rittweger ${ }^{\mathrm{d}, \mathrm{e}}$, Dieter Felsenberg ${ }^{\mathrm{a}}$, \\ Carolyn A. Richardson ${ }^{\text {b }}$
}

${ }^{a}$ Charité Universitätsmedzin Berlin, Zentrum für Muskel- und Knochenforschung, Hindenburgdamm 30, 12200 Berlin, Germany

${ }^{\mathrm{b}}$ School of Health and Rehabilitation Sciences, The University of Queensland, Brisbane, QLD 4072, Australia

'School of Information Technology and Electrical Engineering, The University of Queensland, Brisbane, QLD 4072, Australia

${ }^{\mathrm{d}}$ German Aerospace Center (DLR), Institute of Aerospace Medicine and Space Physiology, Linder Höhe, 51147 Köln, Germany

e Institute for Biomedical Research into Human Movement and Health, Manchester Metropolitan University, M1 5GD Manchester, UK

\section{A R T I C L E I N F O}

Article history:

Received 1 April 2011

Received in revised form 25 August 2011

Accepted 18 September 2011

\section{Keywords:}

Spaceflight

Timing

Signal processing

Phasic

Body composition

Low back pain

\begin{abstract}
A B S T R A C T
To understand the effects of a resistive vibration exercise (RVE) countermeasure on changes in lumbopelvic muscle motor control during prolonged bed-rest, 20 male subjects took part in the Berlin Bed-Rest Study (in 2003-2005) and were randomised to a RVE group or an inactive control group. Surface electromyographic signals recorded from five superficial lumbo-pelvic muscles during a repetitive knee movement task. The task, which required stabilisation of the lumbo-pelvic region, was performed at multiple movement speeds and at multiple time points during and after bed-rest. After excluding effects that could be attributed to increases in subcutaneous fat changes and improvements in movement skill, we found that the RVE intervention ameliorated the generalised increases in activity ratios between movement speeds $(p \leqslant 0.012$ ), reductions in lumbo-pelvic extensor and flexor co-contraction $(p=0.058)$ and increases in root-mean-square electromyographic amplitude $(p=0.001)$ of the lumbar erector spinae muscles. Effects of RVE on preventing increases in amplitude-modulation $(p=0.23)$ of the lumbar erector spinae muscles were not significant. Few significant changes in activation-timing were seen. The RVE intervention during bed-rest, with indirect loading of the spine during exercise, was capable of reducing some, but not all, motor control changes in the lumbo-pelvic musculature during and after bed-rest.
\end{abstract}

(C) 2011 Elsevier Ltd. All rights reserved.

\section{Introduction}

Bed-rest represents a unique model of extreme musculoskeletal disuse, particularly of the lower quadrant (Booth and Gollnick, 1983). The methodology of prolonged bed-rest was originally developed to act as a simulation of the effects of spaceflight on the human body (Nicogossian and Dietlein, 1982). An additional aim of space agencies in implementing bed-rest studies is to better understand the effect of "inactivity" on the human body and in so doing aiming to improve our management of illness on Earth. Specifically in the musculature, the muscle groups most affected by bed-rest are those involved in upright posture and locomotion, such as the triceps surae, vasti and lumbar spine extensors (Belavý et al., 2011, 2009b). In recent works, we have attempted to gain a better understanding of the effects of bed-rest on motor control at the lumbo-pelvic region (Belavý et al., 2010, 2007a,b). Some of the

* Corresponding author at: Charité Universitätsmedizin Berlin, Zentrum für Muskel- und Knochenforschung, Hindenburgdamm 30, D-12203 Berlin, Germany. Tel.: +49 178979 5006; fax: +49307935918.

E-mail address: belavy@gmail.com (D.L. Belavý). findings have included development of generalised overactivity in the superficial lumbo-pelvic muscles, more phasic activation of the lumbar erector spinae and shift to higher median activation frequencies in this same muscle.

However, aside from gaining a better understanding of the effects of bed-rest on the human body, another goal of bed-rest studies is the development of countermeasures against the changes seen in spaceflight simulation. This information will help not only in the development of (exercise) programmes for preventing musculoskeletal deterioration in spaceflight, but may also better insight into treatment regimes on Earth for deconditioned patients. In the Berlin Bed-Rest Study (Armbrecht et al., 2010; Rittweger et al., 2006), a high-load resistive exercise programme with whole-body vibration (RVE) was implemented. This exercise programme was targeted predominately at the bones (Armbrecht et al., 2010; Rittweger et al., 2010) and muscles (Belavý et al., 2009c; Blottner et al., 2006; Mulder et al., 2006) of the lower limbs. Nonetheless, indirect loading of the lumbar spine occurred via shoulder straps. Magnetic resonance imaging investigations in these same subjects showed that the RVE subjects exhibited less atrophy of the short lumbar spine extensor muscles than in the 
control subjects (Belavý et al., 2008). Hence, we hypothesised that the RVE countermeasure would ameliorate the extent of motor control changes, as measured by electromyography, seen in the lumbo-pelvic muscles of the inactive control subjects.

\section{Materials and methods}

\subsection{Bed-rest protocol}

The "Berlin Bed-Rest Study" was implemented by the Centre of Muscle and Bone Research at the Charite Benjamin Franklin Hospital in Berlin, Germany, from February 2003 to May 2005. Twenty male subjects underwent 8 weeks of strict bed-rest with a subsequent 6 -month follow-up recovery period. The bed-rest protocol, as well as inclusion and exclusion criteria, is discussed in detail elsewhere (Rittweger et al., 2006). In brief, subjects were randomly allocated to either a group that remained inactive (controls, CTRL) or a group that underwent a whole-body resistive vibration exercise countermeasure programme (RVE group) using the Galileo Space exercise device (Novotec Medical, Pforzheim, Germany). Subjects were required to remain in bed at all times. Subjects were also requested to restrict movement in bed to the minimal required for hygiene and other necessary daily tasks. Adherence to this protocol was monitored by continuous video recordings and by use of force transducers in the frame of the bed. The institutional ethics committee approved this study and subjects gave their informed written consent. After bed-rest, all subjects were offered a generalised (cardiovascular fitness and strength) rehabilitation programme at a local physiotherapy practice and returned to their normal work and leisure activities.

\subsection{Countermeasure exercise}

RVE subjects underwent two exercise sessions daily (morning and afternoon) of approximately 5-10 min each during bed-rest. A detailed description of the exercise protocol has been published elsewhere (Armbrecht et al., 2010; Rittweger et al., 2006). In brief, whilst in supine position, subjects placed their feet on a suspended vibrating platform. An axial force was placed through the subjects' trunk and spine via elastic shoulder straps. The loading levels applied through the shoulder straps depended upon the exercise manoeuvre performed and the stage of exercise load progression during the study (see Armbrecht et al., 2010; Rittweger et al., 2006 for further detail) and ranged from 1.2 to 1.8 times body weight. A belt was also attached around the pelvis and hand-grips attached to the frame from which the vibrating platform was suspended. Morning and afternoon exercise sessions were performed. The exercises performed were: squats (from $90^{\circ}$ of knee flexion to near full extension), heel raises (with the knees in near extension, heels raised into ankle plantarflexion) and toe raises (with the knees in near extension, forefoot raised into ankle dorsiflexion) against the platform. Each exercise was performed for more than $60 \mathrm{~s}$. In morning sessions, subjects also performed 10 repetitions of "explosive kicks" (explosive pushes against the vibrating platform from near full knee and hip flexion) at intervals of $10 \mathrm{~s}$. The vibration depended upon exercise manoeuvre performed and the stage of exercise progression but ranged from 19 to $26 \mathrm{~Hz}$. However, for each training session the frequency was constant for the duration of each exercise. Vibration frequency was increased if the subject could perform an exercise for more than $100 \mathrm{~s}$. Vibration amplitude was held constant at $4 \mathrm{~mm}$. During afternoon sessions, subjects exercised at only $60-80 \%$ of the static force used in the morning sessions and the exercises were performed continuously until exhaustion.

\subsection{Repetitive knee movement model and testing protocol}

The knee movement and lumbo-pelvic muscle loading model has been described in prior publications (Belavý et al., 2010, $2007 a, b)$. In brief, to allow the examination of motor control parameters in the lumbo-pelvic musculature during isometric activation, a model using repetitive knee movement to stimulate cyclic modulation of isometric lumbo-pelvic muscular activity was conducted. The movement paradigm was implemented using repetitive right knee movement in prone lying. Straps were placed over the subject's buttocks and distal thigh to reduce movement at these points and expedite isometric lumbo-pelvic muscle action. Movement was conducted with the right leg and a spring was attached to the right ankle to counteract the gravitational weight of the lower leg (Richardson and Bullock, 1986; Richardson, 1987). This experimental setup thus permitted standardised loading of the lumbo-pelvic musculature both during and after bedrest.

Subjects conducted right knee flexion and extension between $0^{\circ}$ and $45^{\circ}$ of knee flexion at four movement speeds $(50,75,100$ and 125 cycles per minute [cyc/min]). Three repetitions of $11 \mathrm{~s}$ were conducted at each movement speed. During each repetition, subjects paused their breathing to remove the influence of respiration on lumbo-pelvic muscle activity (Hodges and Gandevia, 2000). Subjects were able to view a feedback monitor through a cut out in the support apparatus. An electrogoniometer was placed at the right knee to provide data on knee position. Baseline data was collected on the first day of bed-rest (BR1). Subsequent testing occurred on the 4th, 13th, 27th, 41st and 53rd days of bed-rest (BR4, BR13, BR27, BR41 and BR53) and on the 3rd, 7th 14th, 28th, 90th, 180th and 360th day of "recovery" $(\mathrm{R}+)$ post-bed-rest $(\mathrm{R}+3, \mathrm{R}+7, \mathrm{R}+14, \mathrm{R}+28, \mathrm{R}+90, \mathrm{R}+180, \mathrm{R}+360)$.

\subsection{Lumbo-pelvic muscle EMG and signal acquisition}

Five superficial lumbo-pelvic muscles were monitored. To examine different functional parts of the erector spinae with surface EMG, electrodes were placed over the lumbar erector spinae with multifidus (LES; at the level of the 5th lumbar vertebrae between the spinous process and a line drawn from the posterior superior iliac spine to the interspace between the 1st and 2nd lumbar vertebrae (de Foa et al., 1989; Ng et al., 2001) and thoracic erector spinae (TES; at the level of the 2nd and 3rd lumbar interspace, $1 \mathrm{~cm}$ medial to a line drawn from posterior superior iliac spine to the lateral border of the erector spinae at the 12th rib (de Foa et al., 1989; Ng et al., 2001). Surface EMG using these placements has been shown to correlate highly with intra-muscular electrodes in the underlying muscles during a range of manoeuvres (Arokoski et al., 1999) and electrodes placed at least $3 \mathrm{~cm}$ apart over different parts of the erector spinae, as in the current work, can be regarded as giving sufficiently specific signals (Vink et al., 1989). The abdominal muscles were monitored with electrodes placed over the internal oblique (IO; the superior electrode placed $1 \mathrm{~cm}$ medial to the anterior superior iliac spine, the inferior electrode placed parallel to the inguinal ligament at the standard inter-electrode distance ( $\mathrm{Ng}$ et al., 1998 ; $\mathrm{Ng}$ et al., 2001)) and external oblique (EO; at the most inferior point of the costal margin orientated along a line from that point to the contralateral pubic tubercle ( $\mathrm{Ng}$ et al., 1998; $\mathrm{Ng}$ et al., 2001)). The inferior portion of the gluteus maximus muscle (IGM), which has a different function to the more cranial portion (Lyons et al., 1983), was monitored with electrodes placed inferior and medial to a line drawn between the posterior superior iliac spine and posterior greater trochanter (Lyons et al., 1983). Bipolar $\mathrm{Ag} / \mathrm{AgCl}$ surface electrodes were placed at an inter-electrode distance of $35 \mathrm{~mm}$. Using the placements utilised in the current work, prior 
work has been able to distinguish functional differences in muscle activation in the different parts of the erector spinae and abdominal obliques (Lyons et al., 1983; Ng et al., 2001, 2002). Electrodes were also placed over the right biceps femoris muscle to monitor leg muscle activity at rest. A ground electrode was placed at the right elbow. Standardised skin preparation was performed involving washing the skin, shaving and the application of an abrasiveconductive gel.

EMG and goniometer data were sampled simultaneously at $2000 \mathrm{~Hz}$ using a Powerlab system running Chart version 4.2 software (AD Instruments, Sydney, Australia) with a 16 bit A/D converter, band-pass filtered from 15 to $500 \mathrm{~Hz}$ and were stored for offline processing. During testing, subjects were given real-time visual feedback on movement speed and position. A second computer also sampled the goniometer signal and implemented custom written software in the Labview environment (version 6.1, National Instruments, Texas) to provide this feedback.

\subsection{Goniometer signal processing and movement accuracy}

Prior to processing EMG data, the goniometer signal, which was sampled simultaneously with the EMG signals, was first processed to select data "regions" which fulfilled the following criteria: beginning at a minima nearest $0^{\circ}$, three consecutive movement cycles during which each movement cycle's speed was within $\pm 5 \mathrm{cyc} /$ min of the target speed, and the maxima (near $45^{\circ}$ ) and minima (near $0^{\circ}$ ) were within $\pm 4^{\circ}$ of their respective targets. This process was conducted to limit the effect of extremes of performance on the observed motor control patterns and further standardise the experiment. This processing also provided information on movement accuracy for correlation analyses: mean-squared-error (MSE) of movement speed ( $\left.M S E_{\text {speed }}\right)$, maxima positions $\left(M S E_{45^{\circ}}\right)$ and minima positions $\left(M S E_{0^{\circ}}\right.$ ). These MSE values were calculated for each data region. In addition, the MSE of movement speed (All-MSE $\left.E_{\text {speed }}\right)$, maxima positions (All-MSE ${ }_{45^{\circ}}$ ) and minima positions $\left(A l l-M S E_{0^{\circ}}\right)$ were calculated for the entire goniometer signal from each trial. This additional movement accuracy data was then used to track changes in movement performance over the course of the study. As we focus here on the motor control changes in bed-rest, data on movement accuracy have been included in online Supplementary material). Unless otherwise stated all algorithms here, were implemented in the Labview environment (version 6.1, National Instruments, Texas).

\subsection{EMG signal processing}

The EMG signal processing method is described in Fig. 1. EMG data were band-pass filtered from 20 to $500 \mathrm{~Hz}$ using a 10th order digital Butterworth filter. Then, in each selected data "region" the corresponding EMG signal was extracted and the rootmean-square (RMS) activation level was computed. In addition to this, the following variables were calculated:

- Ratio: the calculation of EMG amplitude "ratios" between experimental conditions have been used previously to examine the effects of different movement protocols (Hodges and Gandevia, 2000) and have been shown to reduce inter-subject variability (Winter and Yack, 1987). As it was inappropriate, and difficult practically in the horizontal position, to perform maximal voluntary contractions repeatedly during a strict bed-rest study, the use of amplitude-ratios between movement speeds helps to avoid some of the difficulties associated with measuring raw EMG amplitude (such as due to subcutaneous fat; Nordander et al., 2003). In the current work, we calculated three "activity-ratios" $\left(\right.$ ratio $_{75 / 50}$, ratio $_{100 / 75}$, ratio $_{125 / 100}$ ) for each muscle by dividing each RMS value from movement speeds 75 cyc/min and above by the median RMS value of the speed below. This variable could be considered a measure of the degree to which the central nervous system "ramps" the activity-levels of each muscle with increases in movement speed.

- CoCon: abdominal flexor-lumbar extensor antagonistic cocontraction (CoCon) was quantified using an "area-normalisation" method adapted from prior work (Vinther et al., 2005; Winter, 2004). The following algorithm was used:

a. Generation of a "linear-envelope" from the entire $11 \mathrm{~s}$ EMG signals of the EO, IO, LES and TES muscles by highpass filtering at $100 \mathrm{~Hz}$ using a digital 10th order quasiButterworth filter, rectification, and then low-pass filtering using a $10 \mathrm{~Hz}$ digital 10th order Bessel filter.

b. Partitioning of the linear-envelope into the appropriate data region (as determined from goniometer signal analysis).

c. Normalisation of the area of each linear-envelope region to " 1 " by dividing each value by the total area under the curve.

d. The greater value of the LES and TES signals was taken at each data point and the resulting signal was re-normalised as in step [c] (generating an "extensor linearenvelope").

e. The abdominal IO and EO signals were processed in the same fashion as in steps [b], [c] and [d] to yield a "flexor linear-envelope".

f. The area of overlap of the flexor and extensor linear-envelopes was calculated and this value ranged from 0 to 1 and represented the lumbo-pelvic flexor and extensor co-contraction variable (CoCon).

- BTR: or "burst-to-tonic ratio" examined the degree of amplitude-modulation present in the EMG signal. This measurement aimed to quantify the extent of a phasic "burst" of electromyographic activity superimposed on the ongoing, or "tonic", activity during the movement cycle (Belavý et al., 2009a,b). Since the muscles were typically continuously active during the movement task, the following algorithm was used:

a. Generation of a "linear-envelope" from the entire $11 \mathrm{~s}$ EMG signal of every muscle (as in step [a] of CoCon calculation: $100 \mathrm{~Hz}$ high-pass, 10th order digital Butterworth filter, followed by full-wave rectification, and then low pass filtering using a $10 \mathrm{~Hz}, 10$ th order digital Bessel filter).

b. Partitioning of the linear-envelope into the appropriate data region (as per step [b] of CoCon calculation).

c. Detection of maxima and minima of the linear-envelope using quadratic least-squares fit.

d. Calculation of median maximum and median minimum values.

e. Calculation of the ratio of the median maximum to minimum values, giving the "burst-tonic-ratio" (BTR) representing the relative height of the phasic burst component to the underlying ongoing tonic EMG activity. Thus, BTR values approaching "1" indicate more tonic muscle activation.

Due to the number of parameters examined, we focus on the ratio, CoCon and BTR variables in the current publication as these variables, in our view, are most functionally relevant and resistant to extraneous factors, such as subcutaneous fat. However, for the interested reader, we include in online Supplementary material the data on the RMS variables. We also examined the timing of muscle activity using an algorithm implemented in previous works (Belavý et al., 2010; Belavý et al., 2006). These latter data have been presented in online Supplementary data for the interested reader. 


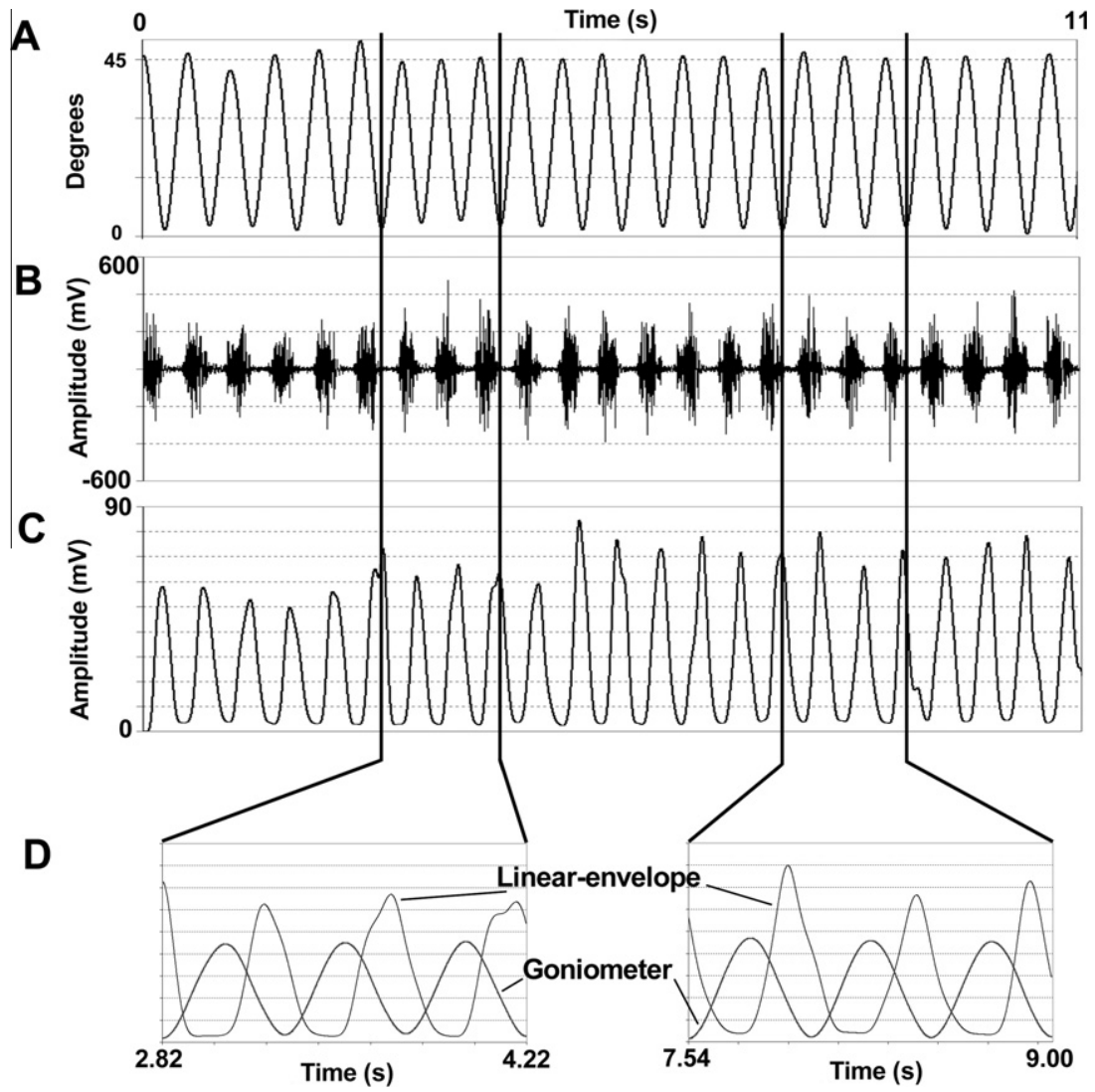

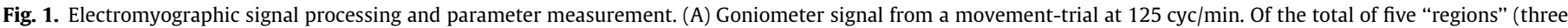

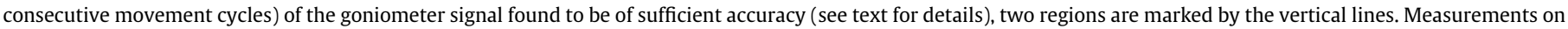

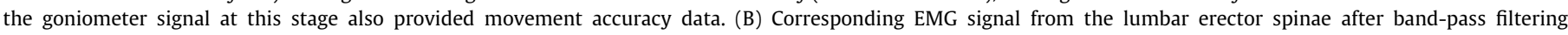

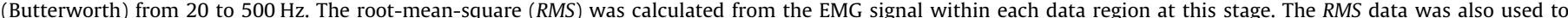

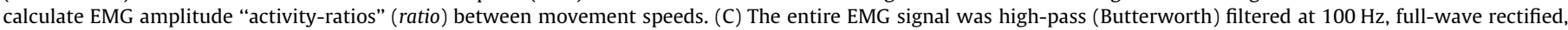

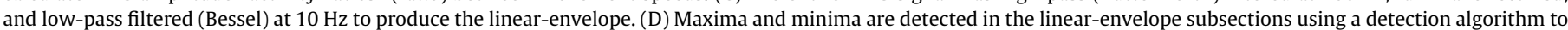

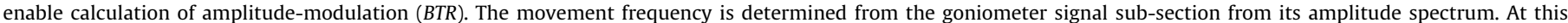

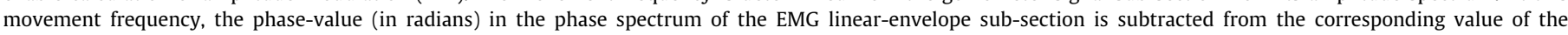

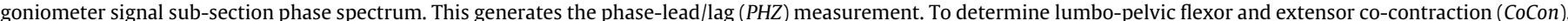

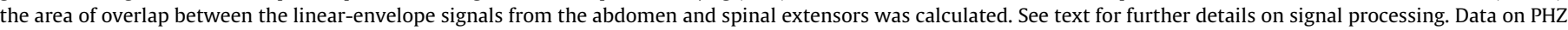
and RMS have been presented in online Supplementary material for the interested reader and are not discussed in detail here.

\subsection{Body composition data}

A Delphi W (Hologic, Waltham, MA) system was used to perform total body scans according to the standard Hologic Operator's Manual 3-days prior to bed-rest (BDC-3), on day BR2, BR17, BR31, BR45 and BR55 of the bed-rest phase and on $R+14, R+28, R+90$, $R+180$ and $R+360$ during recovery phase. Fat mass (in kilograms) of the trunk (from pelvis to shoulders) were derived from the whole-body scan. All scanning and analyses were performed by the same operator to ensure consistency and standard quality control procedures were followed. BDC-3 and BR2 data were averaged prior to provide a more precise baseline measure.

\subsection{Further data processing and statistical analyses}

The natural-logarithm of the ratio and BTR variables was calculated prior to further analysis in order to approximate a near-normal distribution of the residuals. For the EMG variables ratio and BTR linear mixed-effects models (Pinheiro, 2000) were used to fit fixed effects for "group", "muscle", "study-date", "movement speed" and all interactions up to a four-way interaction between these variables. For the CoCon and All-MSE variables similar models were constructed but without the factor of "muscle". Similarly, for trunk fat mass, factors of "group", "study-date" and their two-way interaction were included in the calculation. Differences between the groups at baseline were also evaluated in a similar fashion, but without the "study-date" term. Allowances for heterogeneity of variance were made due to "group", "muscle", "study-date" and/or "movement speed" where necessary. Depending on the results from analysis of variance (ANOVA), subsequent modelling was performed as appropriate, such as between groups within each muscle or within movement speeds to determine which level of a given factor was responsible for an effect. Subsequently, the changes within each group compared to baseline were calculated. An $\alpha$ of 0.05 was taken for statistical significance on ANOVA. As multiple measurement sessions were undertaken on the same subjects, we examined for consistent significant differences across testing days. Correlation (Pearson) analyses, using the baseline data alone, were also performed between the movement accuracy and EMG variables and also between trunk fat mass and EMG variables (presented in online Supplementary material). For the movement accuracy and EMG variables, partial (Pearson) correlation analyses, controlling for muscle and movement speed, were also performed (online Supplementary material). The "R" statistical environment (version 2.10.1, www.r-project.org) was used to implement analyses. 


\section{Results}

Due to subject absence or technical difficulties, not all data from each subject on every scheduled testing day were available. The numbers of subjects able to be included in statistical analysis on each testing day are given in online Supplementary material.

\subsection{Baseline data and correlation analyses}

Data from the first day of bed-rest (BR1) are presented in Tables 1 and 2 . There were no differences between the groups at baseline (Tables 1 and 2). Correlations amongst movement accuracy and electromyographic variables as well as partial correlations (controlling for muscle and movement speed) are presented in online Supplementary material. When the influence of muscle and movement speed are not controlled for, a number of low to moderate correlations exist between movement accuracy and EMG variables and also between each of the EMG variables. The partial correlations (online Supplementary material) show, however, that there was little relation between the movement accuracy variables and the EMG parameters. However, some moderate correlations between EMG parameters persisted.

\subsection{Movement accuracy}

Performance of the movement task, as measured by meansquared-error of the goniometer signal maxima (around the $45^{\circ}$ target; $A l l-M S E_{45^{\circ}}$ ) and minima (around the $0^{\circ}$ target; All-MSE $0^{\circ}$ ) as well as movement speed (All-MSE $\left.E_{\text {speed }}\right)$ typically improved over the course of the study (online Supplementary material). This effect was strongest in the All-MSE $45^{\circ}$ and $A l l-M S E_{\text {speed }}$ variables with reductions in both of these variables seen in both groups (online Supplementary material). The improvements in movement performance did not differ between the RVE and CTRL groups for All-MSE ${ }_{45^{\circ}}$ or All-MSE $E_{\text {speed }}(p \geqslant 0.09)$. For All-MSE $E_{0^{\circ}}$ there was some evidence of a different response between the two groups (group $\times$ study-date $\times$ movement speed: $p=0.006$ ), though further ANOVAs at each movement speed suggested a difference between the groups at the $75 \mathrm{cyc} / \mathrm{min}$ movement speed only $(p=0.045$;
Table 1

Baseline (BR1) flexor-extensor co-contraction.

\begin{tabular}{lll}
\hline $\begin{array}{l}\text { Movement } \\
\text { speed }\end{array}$ & CoCon \\
\cline { 2 - 3 } & $\begin{array}{l}\text { Inactive control group } \\
\text { (CTRL) }\end{array}$ & $\begin{array}{l}\text { Resistive vibration exercise } \\
\text { group (RVE) }\end{array}$ \\
\hline $50 \mathrm{cyc} / \mathrm{min}$ & $0.91(0.02)$ & $0.92(0.02)$ \\
$75 \mathrm{cyc} / \mathrm{min}$ & $0.88(0.02)^{*}$ & $0.86(0.02)^{*}$ \\
$100 \mathrm{cyc} / \mathrm{min}$ & $0.82(0.02)^{\ddagger}$ & $0.77(0.02)^{\ddagger}$ \\
$125 \mathrm{cyc} / \mathrm{min}$ & $0.80(0.02)^{\ddagger}$ & $0.73(0.03)^{\ddagger}$
\end{tabular}

CoCon variable (lumbo-pelvic extensor and flexor co-contraction) does not have a unit. ${ }^{*} p<0.05$. ${ }^{\dagger} p<0.01 .{ }^{\star} p<0.001$ and indicates significance of difference to value at $50 \mathrm{cyc} / \mathrm{min}$. Reductions in the CoCon variable imply less overlap between extensor and flexor muscle activity and hence less co-contraction. There were no differences between the groups at baseline $(p=0.072)$.

otherwise $p \geqslant 0.10)$. At this $75 \mathrm{cyc} / \mathrm{min}$ speed, no significant effects changes compared to baseline were seen in either group and there was no clear pattern of difference between the two groups (data not shown).

\subsection{Electromyographic variables}

For the ratio variable, significant group $\times$ study-date $(p=0.011)$ and group $\times$ study-date $\times$ movement speed $(p=0.012)$ effects were seen on ANOVA, with otherwise no significant differences between muscles over time $(p \geqslant 0.58)$. Pooled across all movement speeds, increases in amplitude-ratios were seen in the CTRL group, with decreases in the RVE group (Fig. 2). When examining the ratio data at each movement speed (Fig. 3), the increased activity-ratios were seen in the CTRL group at the highest speeds ( ratio $_{125 / 100}$ ), whereas RVE group shows little change at highest movement speeds, but reduction of ratio $_{75 / 50}$ over the course of study. The different responses of the two groups were significant for ratio $0_{75 / 50}$ $(p=0.0068)$ and ratio $_{125 / 100}(p=0.0036)$ but not for ratio ${ }_{100 / 75}$ $(p=0.26)$.

Lumbo-pelvic extensor and flexor co-contraction (CoCon) decreased in the CTRL group with typically non-significant increases in the RVE group (Fig. 2). The difference between the groups did not however reach significance on ANOVA (group $\times$ study-date:

Table 2

Baseline (BR1) electromyographic parameters.

\begin{tabular}{|c|c|c|c|c|c|}
\hline \multirow[t]{2}{*}{ Movement speed (cyc/min) } & \multicolumn{5}{|l|}{ Muscle } \\
\hline & External oblique & Inferior gluteus maximus & Internal oblique & Lumbar erector spinae & Thoracic erector spinae \\
\hline \multicolumn{6}{|c|}{ Amplitude-ratio (no unit) - inactive control group (CTRL) } \\
\hline 75 & $1.26(0.13)$ & $1.37(0.21)$ & $1.57(0.21)$ & $2.40(0.54)$ & $1.27(0.17)$ \\
\hline 100 & $1.65(0.17)$ & $1.80(0.28)$ & $1.83(0.25)$ & $2.15(0.48)$ & $1.54(0.21)$ \\
\hline 125 & $1.69(0.20)$ & $1.88(0.31)$ & $1.43(0.21)$ & $1.59(0.38)$ & $1.61(0.24)$ \\
\hline \multicolumn{6}{|c|}{ Amplitude-ratio (no unit) - resistive vibration exercise group (RVE) } \\
\hline 75 & $1.17(0.14)$ & $1.26(0.07)$ & $1.62(0.17)$ & $4.14(0.57)$ & $1.29(0.14)$ \\
\hline 100 & $1.50(0.18)$ & $1.51(0.09)$ & $1.77(0.18)$ & $2.81(0.38)$ & $2.30(0.25)^{\ddagger}$ \\
\hline 125 & $1.58(0.23)$ & $1.82(0.14)^{\dagger}$ & $1.67(0.21)$ & $1.65(0.28)^{\dagger}$ & $1.65(0.21)$ \\
\hline \multicolumn{6}{|c|}{ Amplitude-modulation (BTR; no unit) -- inactive control group (CTRL) } \\
\hline 50 & $1.46(0.13)$ & $1.34(0.15)$ & $1.55(0.07)$ & $1.45(0.33)$ & $1.39(0.12)$ \\
\hline 75 & $1.60(0.15)$ & $1.70(0.19)$ & $1.62(0.08)$ & $2.53(0.57)^{*}$ & $1.56(0.14)$ \\
\hline 100 & $1.79(0.17)$ & $2.33(0.26)^{\ddagger}$ & $1.75(0.08)^{\dagger}$ & $5.47(1.24)^{\ddagger}$ & $2.01(0.19)^{\dagger}$ \\
\hline 125 & $2.55(0.26)^{\ddagger}$ & $2.90(0.35)^{\ddagger}$ & $1.98(0.10)^{\ddagger}$ & $6.23(1.49)^{\ddagger}$ & $2.38(0.24)^{\ddagger}$ \\
\hline \multicolumn{6}{|c|}{ Amplitude-modulation (BTR; no unit) -- resistive vibration exercise group (RVE) } \\
\hline 50 & $1.44(0.05)$ & $1.29(0.09)$ & $1.51(0.14)$ & $1.30(0.26)$ & $1.34(0.13)$ \\
\hline 75 & $1.45(0.05)$ & $1.59(0.12)^{*}$ & $1.60(0.15)$ & $3.53(0.74)^{\dagger}$ & $1.54(0.15)$ \\
\hline 100 & $1.59(0.06)^{*}$ & $2.05(0.15)^{\ddagger}$ & $1.89(0.17)^{*}$ & $8.06(1.66)^{\ddagger}$ & $2.30(0.23)^{\dagger}$ \\
\hline 125 & $1.80(0.09)^{\dagger}$ & $2.34(0.21)^{\star}$ & $2.00(0.22)^{*}$ & $14.43(3.50)^{\ddagger}$ & $2.59(0.33)^{\ddagger}$ \\
\hline
\end{tabular}

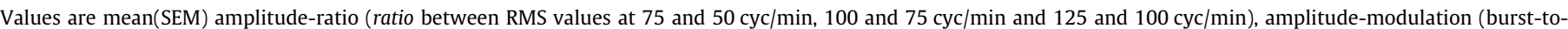

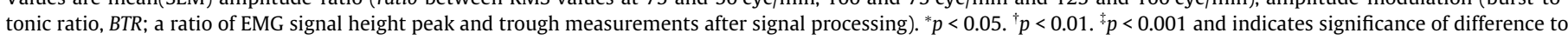

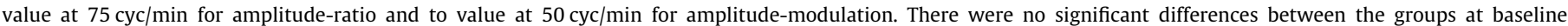
$(p \geqslant 0.096)$. See Table 1 for baseline values of lumbo-pelvic extensor and flexor co-contraction (CoCon). 


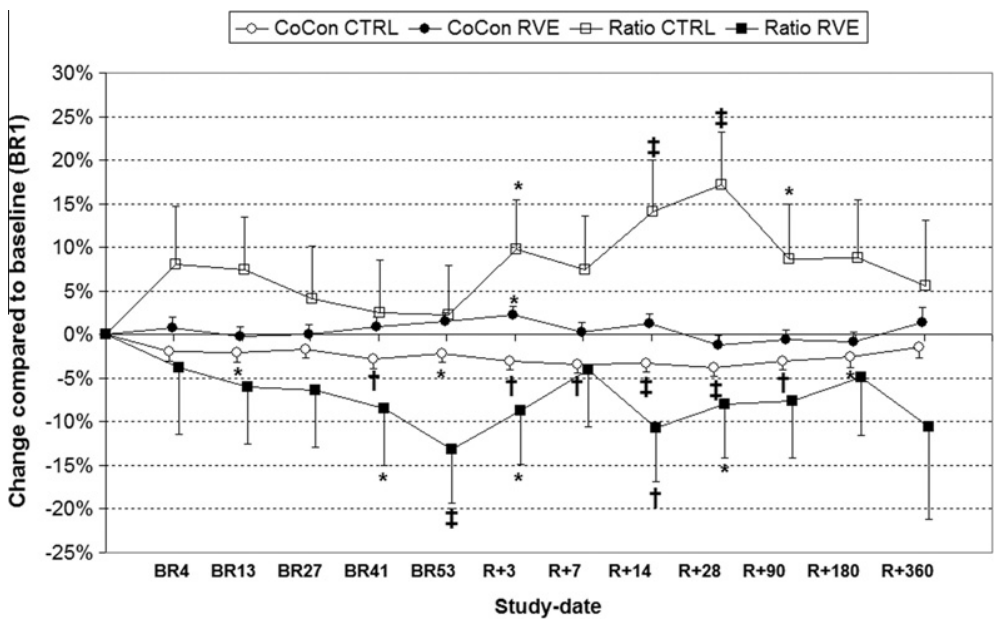

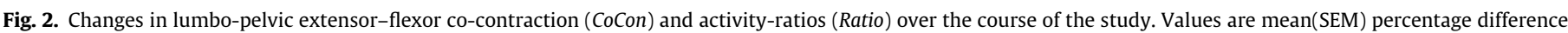

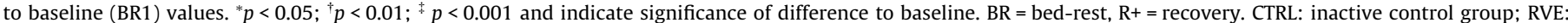

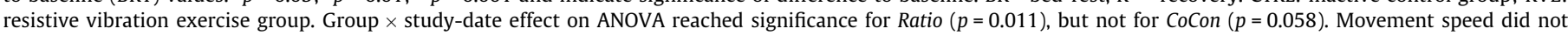

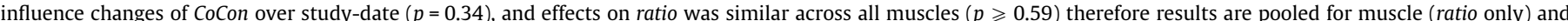

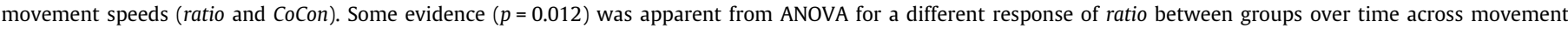
speeds; see Fig. 3.
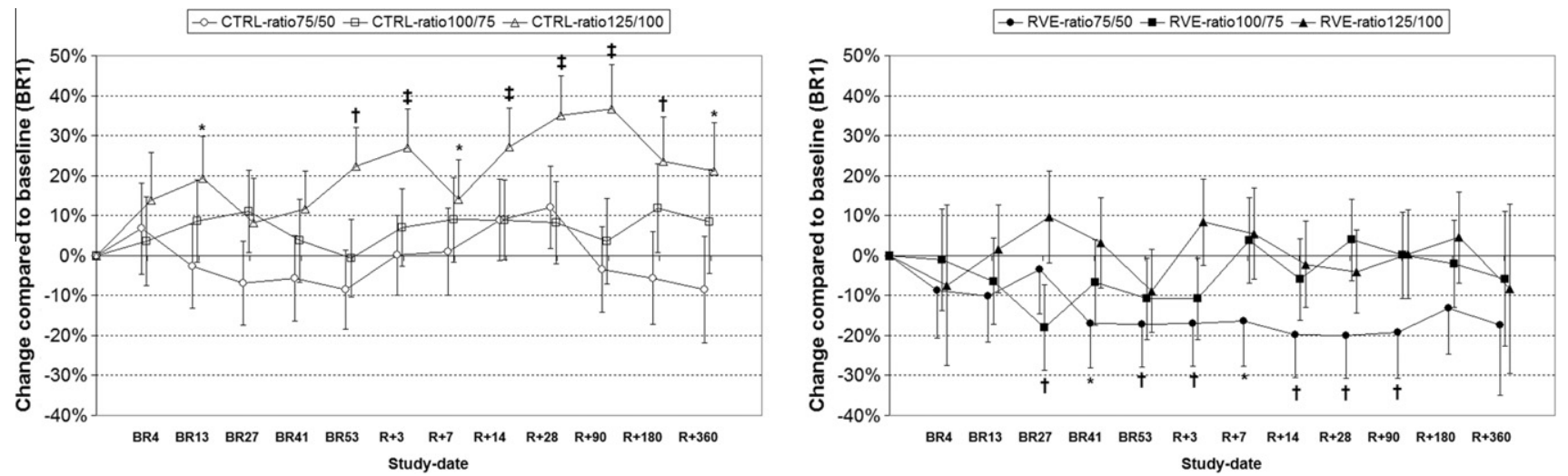

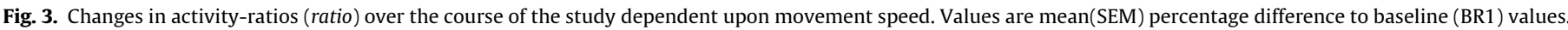

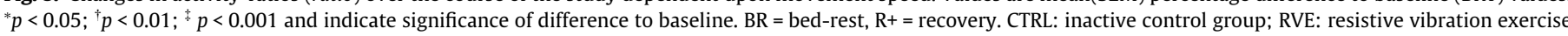

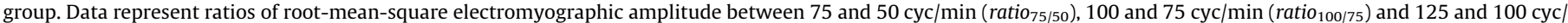

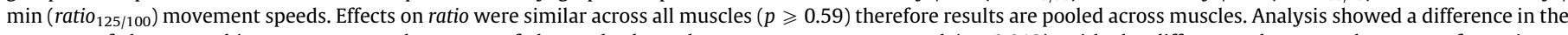

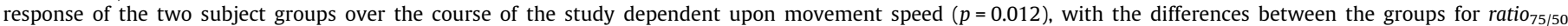
$(p=0.0068)$ and ratio $_{125 / 100}(p=0.0036)$ reaching significance (otherwise $\left.p=0.26\right)$.

$p=0.058)$. No influence of movement speed on changes over time ( $p=0.34$ ) were seen for the CoCon variable.

Changes in amplitude-modulation (BTR) differed across muscles during the study (study-date $\times$ muscle: $p=0.0039$ ), with different response seen in the different groups (group $\times$ study-date $\times$ muscle: $p=0.0126)$ with no influence of movement speed in these effects $(p \geqslant 0.70)$. Therefore the data presented in Fig. 4 have been pooled across movement speeds. The EO muscle showed no consistent pattern of change in either group (group $\times$ study-date: $p=0.11$ ). The IO muscle showed marginal increases of BTR in both groups, though there were no differences between the two groups (group $\times$ study-date: $p=0.43$ ). The LES muscles showed significant increases in BTR in the CTRL group, and whilst such increases were not seen in the RVE group, the difference between the two groups was not significant on ANOVA (group $\times$ study-date: $p=0.23$ ). BTR in the TES muscle group showed little significant change in the CTRL group, but increases seen in the RVE group, but there were no significant differences between the two groups on ANOVA (group $\times$ study-date: $p=0.55$ ). The IGM muscle showed non- significant decreases in the CTRL group but significant increases in the RVE group with this effect being significant on ANOVA (group $\times$ study-date: $p=0.001$ ).

\subsection{Trunk fat mass}

Baseline mean(SEM) trunk fat mass was $7.15(1.20) \mathrm{kg}$ in the CTRL group and 9.34(1.24) kg in the RVE group with no significant difference between the two groups at baseline $(p=0.21)$. Strongly significant increases in trunk fat mass were seen in the CTRL group during and after bed-rest (Fig. 5) with weaker increases in the RVE group. This effect was not significant on ANOVA, however (group $\times$ study-date: $p=0.19$ ). Correlations between baseline trunk fat mass (average of BDC-3 and BR2) and EMG (BR1) variables are shown in online Supplementary material. A consistent pattern of correlation was however not seen between trunk fat mass and the BTR or ratio variables and there was no association between trunk fat mass and CoCon. 

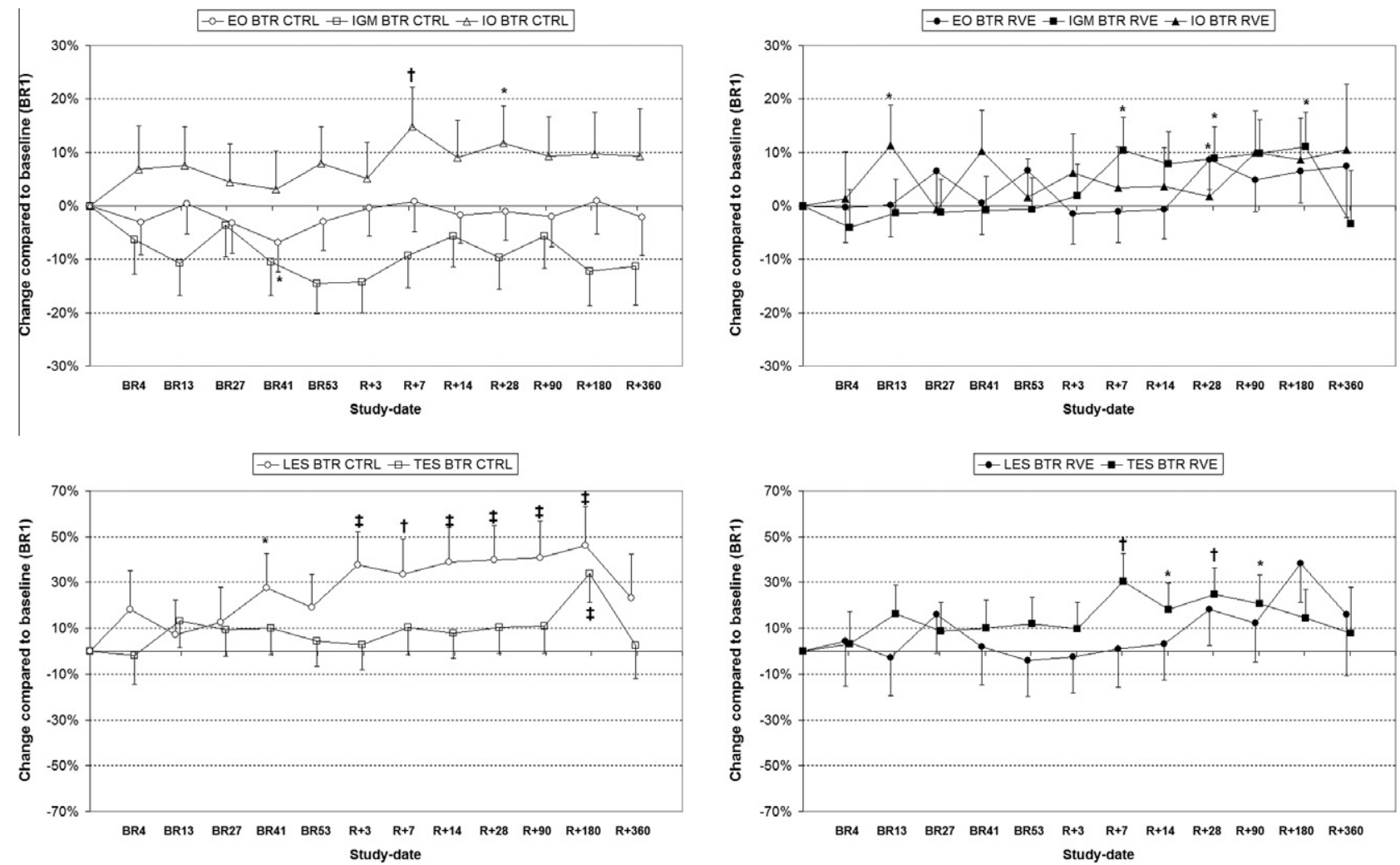

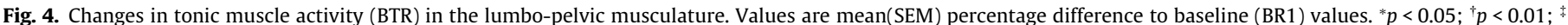

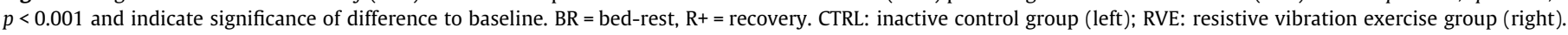

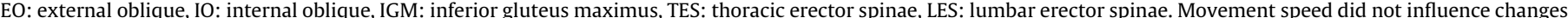

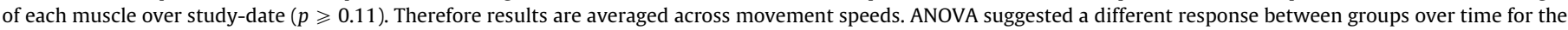

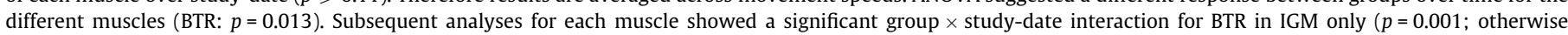
$p \geqslant 0.113)$

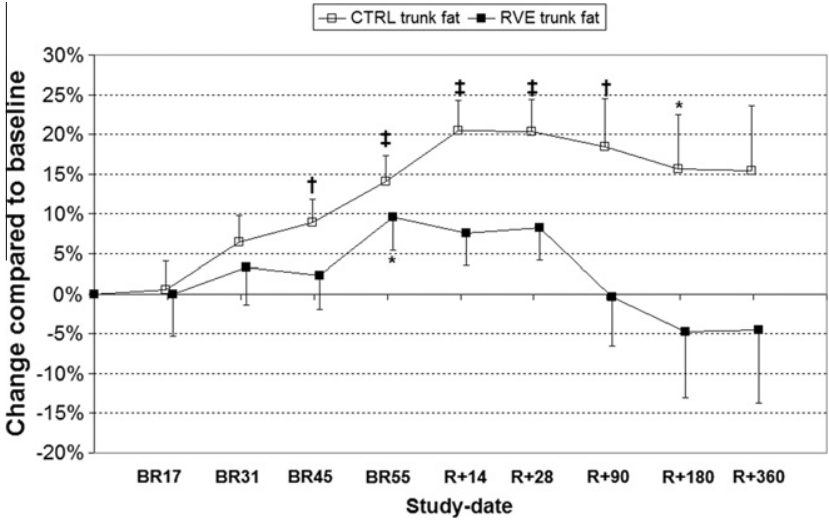

Fig. 5. Changes in trunk fat mass as measured by dual X-ray absorptiometry. Values are mean(SEM) percentage difference to baseline values. Baseline values were average of data collected 3-days prior to beginning of bed-rest (BDC-3) and on day3 of bed-rest (BR2). ${ }^{*} p<0.05 ;{ }^{\dagger} p<0.01 ;{ }^{\dagger} p<0.001$ and indicate significance of difference to baseline. $\mathrm{BR}=$ bed-rest, $\mathrm{R}+=$ recovery. $\mathrm{CTRL}$ : inactive control group; RVE: resistive vibration exercise group. Although the CTRL group showed stronger increases in trunk fat levels than the RVE group, this effect was not statistically significant on ANOVA (group $\times$ study-date: $p=0.19$ ).

\section{Discussion}

The current study examined the effect of a resistive vibration exercise (RVE) countermeasure on preventing motor control changes in the lumbo-pelvic musculature due to 56 days strict bed-rest. The loading of the spine during this exercise countermea- sure occurred indirectly via shoulder straps. A number of differences were observed between the two subject groups, although not all were significant statistically on analysis of variance. These effects included an increase in activity-ratios (or "ramping" of electromyographic activation levels between movement speeds) in the inactive CTRL group but decreases in the RVE group which was significant on analysis of variance. Although the effects did not reach significance, the RVE group did not show the reductions of lumbopelvic extensor and flexor co-contraction seen in the CTRL group and the strong increase of lumbar erector spinae amplitude-modulation was not seen in the RVE group. Few changes were seen in the electromyographic signals from the thoracic erector spinae muscle group, with also little change of amplitude-modulation in the external oblique and internal oblique muscles. Prior publications (Belavý et al., 2010, 2007a,b) have considered the effects in the CTRL group in detail and thus the current discussion focuses on the impact of the exercise countermeasure using the CTRL group data as a reference only. The first step in understanding the true impact of the exercise countermeasure on lumbo-pelvic motor control during bed-rest is to determine which effects could be due to confounding factors, such as improvements in motor performance and alterations in subcutaneous fat levels.

As the subcutaneous fat layer increases in size, there is a greater low-pass filtering effect on the EMG signal and hence there is an alteration of the measured signal properties (de Luca, 1997). Since bed-rest typically results in changes in body composition (Blanc et al., 1998), we measured trunk fat mass using whole-body dual X-ray absorptiometry to examine whether this may represent a confounding factor for our results. Our results, similar to those of prior work (Hemingway et al., 1995; Nordander et al., 2003), 
showed a negative correlation between trunk fat mass and electromyography activity-levels (online Supplementary material), but the relationships to other electromyography variables was less consistent. Hence, the effects seen in the amplitude-modulation, lumbo-pelvic extensor and flexor co-contraction and amplituderatio variables are unlikely to be due to increases in fat mass.

It is also possible that the improvements in movement performance seen over the course of the study contributed to the changes seen in motor control. In the partial-correlation analyses performed, little relationship could be found between movement accuracy and the electromyographic parameters. Although no clear relationship was seen, this of course does not rule out that skill acquisition may have played a role. Indeed, in considering a number of studies on changes in electromyographic parameters with skill acquisition, the relationship between skill improvement and motor control changes is not always clear. Different studies, often using different motor tasks, showed differing patterns of motor control adaptation with practice (Bernardi et al., 1996; Carson and Riek, 2001; Gabriel and Boucher, 1998; Gentili et al., 2006; Gribble et al., 2003; Ludwig, 1982), implying that the effect is highly dependent upon the task and muscle considered. Some authors also pointed out that motor control change with practice was often subject specific (Carson and Riek, 2001). Consequently, although we found no correlation between movement accuracy and the electromyographic parameters measured, it cannot be ruled out that skill improvement did not cause some of the changes seen in motor control, and a future longitudinal-study of skill acquisition with the current motor task would be required to resolve this issue. Nonetheless, the two subject groups in the current study showed similar improvements in motor skill over the course of the study. Hence, where the two groups showed opposing changes in motor control, it is likely these effects were due to an impact of the countermeasure, rather than practice.

In this vein, this leaves the effects on activity-ratios (increased in CTRL, decreased in RVE), lumbo-pelvic extensor and flexor cocontraction (decreased in CTRL, marginal increases in RVE) and amplitude-modulation in the lumbar erector spinae (greater increases in CTRL than RVE) as effects which could be attributed to the exercise countermeasure. Changes in trunk fat levels and movement accuracy cannot explain these findings and we consider that these effects were indeed due to a treatment effect of the RVE countermeasure. It should be stressed that the differences between groups in co-contraction represented a trend and the effect on lumbar erector spinae amplitude-modulation were not significant. The lumbar erector spinae muscle group showed the greatest number of changes in motor control due to bed-rest (Belavý et al., 2010, 2007a) and magnetic resonance imaging studies in the same subjects showed that this muscle group also showed the greatest amount of atrophy of the lumbo-pelvic muscles (Hides et al., 2007). The RVE countermeasure also reduced cross-sectional area loss of these short spinal extensors (Belavý et al., 2008). Thus, it is quite plausible that the amelioration of the motor control changes seen in the lumbar erector spinae muscle group was indeed a result of the countermeasure. It should be remembered that the movement task performed required the muscles of the lumbopelvic region to supply a "stable base" to permit efficient knee movement during the movement task. In light of this, given that the exercise countermeasure reduced losses in the "deeper" muscles of the lumbo-pelvic region (Belavý et al., 2008), it is our view that the increased "ramping" of superficial muscle activity-levels (as measured by amplitude-ratios) during the movement task was not necessary in the RVE group to stabilise the lumbo-pelvic region. The reduction of extensor-flexor co-contraction in the CTRL group could well be considered to be a reduction in motor co-ordination at the lumbo-pelvic region (Belavý et al., 2007b), which was ameliorated (trend) by the RVE countermeasure.

Some of the findings from the inferior portion of the gluteus maximus muscle cannot be explained so readily. Significant increases of amplitude-modulation were seen for this muscle in the RVE group after bed-rest with little change in the CTRL group. Magnetic resonance imaging measurements (Grimaldi, 2008) of inferior gluteus maximus muscle cross-sectional area in the same subjects showed similar losses in both groups, on average $15.6 \%$. It should be noted that in this magnetic resonance imaging study, only six subjects were measured in each group. Also, of the muscles measured, inferior portion of the gluteus maximus muscle was the most difficult from which to get strong electromyographic signal (due to the thick layer of overlying buttock-fat tissue). Further work would need to be performed to better understand the findings in this muscle.

It would be appropriate to discuss some of the mechanisms by which the RVE countermeasure may have reduced the motor control changes at the lumbo-pelvic region during and after bed-rest. On the one hand, the mechanical loading due to resistive exercise during exercise no doubt acted as a stimulus to maintain the musculature and also baseline neuromuscular function. However, whole-body vibration however can modulate lumbo-pelvic neuromuscular control, at least transiently (e.g. Fontana et al., 2005). Due to logistical and financial restrictions, the current study did not have an additional group with either only resistive exercise or only vibration exercise which would have helped to understand the effect of whole-body vibration alone. Hence, the reader should be cautioned against interpreting the findings of the current study on the RVE countermeasure as an effect of vibration per se: the countermeasure could have had an impact upon lumbo-pelvic muscle function via a number of physiological pathways.

The effects seen in the training group have some exercise prescription implications. Firstly, in terms of countermeasure exercise for spaceflight (simulation), effects on preventing motor control change at the lumbo-pelvic region can be achieved despite not including specific exercise manoeuvres for certain muscle groups (such as the extensors): in the current study indirect loading of the spine occurred via shoulder straps and the subjects needed to control their spinal position under this load. Whilst we argue that high-load resistive exercise is important for maintaining muscle mass in spaceflight (and simulation; see Belavý et al., 2009c for discussion), the maintenance of motor control and inter-muscular co-ordination appears to be more sensitive, even to lower loads. It should be noted however, that the subjects of the current study were young healthy men and caution is advised before applying such exercise protocols in patient populations (e.g. low back pain).

It is also important to mention some of the limitations of the current study. As in almost all studies in real or simulated microgravity the sample size in this study is also small, which may have prevented some effects from reaching statistical significance. The small number of subjects available for testing one-year after bedrest may well have skewed the mean on this testing day. As already stressed, it is also important to note that further study should examine the effect of vibration alone or resistive exercise alone on the parameters measured here. Due to cost limitations in bedrest studies, the Berlin Bed-Rest Study aimed first to evaluate the effectiveness of the countermeasure program as a whole with further studies to be planned should the countermeasure prove effective. As the current investigation was the first of its kind in prolonged bed-rest we unfortunately do not have access to prior data, or data on measurement repeatability, upon which to perform a sensitivity-analysis. Such a sensitivity-analysis would aid in the interpretation of non-significant findings. Also, future work 
could consider whether additional benefits could be gained by other forms of exercise on preventing the effects of bed-rest on the parameters measured here. Additionally, it should be noted that some electromyographic parameters are related. Whilst the electromyographic parameters were designed to quantify different aspects of muscle function, there was some overlap between parameters. This effect was most obvious for root-mean-square electromyographic amplitude and amplitude-modulation where the partial-correlation analyses (online Supplementary material) showed a weak to moderate correlation between these two variables at baseline and the changes over time in these two parameters largely paralleled one another. For the remaining electromyographic parameters, however, the correlations between them were quite weak, indicating that they do indeed measure different aspects of motor control.

In conclusion, the current study examined the effect of a resistive vibration exercise (RVE) countermeasure on preventing motor control changes in the lumbo-pelvic musculature due to 56 days strict bed-rest. After considering the potential confounding influences of increases in trunk fat levels and improvements in motor skill, we found a significant impact of the RVE intervention in ameliorating the generalised increases in activity-ratios of the lumbopelvic musculature seen in bed-rest. Furthermore, the RVE group showed marginal increases lumbo-pelvic extensor and flexor cocontraction whereas this reduced significantly in the CTRL group, although this between-group effect was trend and did not reach significance. Also, although the between-group effects were not statistically significant, the RVE group also did not show the increases in amplitude-modulation of the lumbar erector spinae that were observed in the CTRL group. These results showed that the RVE countermeasure as implemented was capable of ameliorating some, but not all, changes of motor control in the lumbo-pelvic region as a result of prolonged bed-rest.

\section{Conflict of interest statement}

The authors have no conflicts of interest.

\section{Acknowledgements}

The authors wish to thank the subjects who participated in the study, and the staff of the Center of Muscle and Bone Research at the Charité Campus Benjamin Franklin Hospital, Berlin, Germany who helped implement the bed-rest study. The lead author wishes to thank Anne Diepgen, Björn Bühring and Franzi Luhn for their support during the lengthy data collection period. The IT-staff of the School of Information Technology and Electrical Engineering (Brisbane) are thanked for access to high-speed computing facilities to perform signal processing and statistical modelling. The Berlin Bed-Rest Study was supported by grant 14431/ 02/NL/SH2 from the European Space Agency. The Berlin Bed-Rest Study was also sponsored by the Charité Campus Benjamin Franklin, DLR (German AeroSpace), MSD Sharp \& Dohme, Lilly Germany, Servier Germany, Hoffmann-LaRoche, Siemens, Novartis, Danone and Seca. The writing of this publication was supported in part by grant number FE 468/5-1 from the German Research Foundation (DFG) and grant number 50WB0720 from the German AeroSpace Center (DLR).

\section{Appendix A. Supplementary data}

Supplementary data associated with this article can be found, in the online version, at doi:10.1016/j.jelekin.2011.09.009.

\section{References}

Armbrecht G, Belavý DL, Gast G, Bongrazio M, Touby F, Beller G, et al. Resistive vibration exercise attenuates bone and muscle atrophy in 56 days of bed rest: biochemical markers of bone metabolism. Osteoporos Int 2010;21(4):597-607.

Arokoski JP, Kankaanpaa M, Valta T, Juvonen I, Partanen J, Taimela S, et al. Back and hip extensor muscle function during therapeutic exercises. Arch Phys Med Rehabil 1999;80(7):842-50.

Belavý DL, Wilson SJ, Richardson CA. Quantification of the timing of continuous muscle activity in a repetitive-movement task. Physiol Meas 2006;27(11):1143-50.

Belavý DL, Richardson CA, Wilson SJ, Felsenberg D, Rittweger J. Tonic to phasic shift of lumbo-pelvic muscle activity during 8 -weeks of bed-rest and 6-months follow-up. J Appl Physiol 2007a;103(1):48-54

Belavý DL, Richardson CA, Wilson SJ, Rittweger J, Felsenberg D. Superficial lumbopelvic muscle overactivity and decreased co-contraction after 8-weeks of bedrest. Spine 2007b;32(1):E23-9.

Belavý DL, Hides JA, Wilson SJ, Stanton W, Dimeo FC, Rittweger J, et al. Resistive simulated weightbearing exercise with whole body vibration reduces lumbar spine deconditioning in bed-rest. Spine 2008;33(5):E121-31.

Belavý DL, Mehnert A, Wilson S, Richardson CA. Analysis of burst and tonic electromyography characteristics in a repetitive-movement task: electromyographic simulation and comparison of novel morphological and linear-envelope approaches. J Electromyogr Kinesiol 2009a;19(1):10-21.

Belavý DL, Miokovic T, Armbrecht G, Richardson CA, Rittweger J, Felsenberg D. Differential atrophy of the lower-limb musculature during prolonged bed-rest. Eur J Appl Physiol 2009b;107(4):489-99.

Belavý DL, Miokovic T, Armbrecht G, Rittweger J, Felsenberg D. Resistive vibration exercise reduces lower limb muscle atrophy during 56-day bed-rest. J Musculoskelet Neuronal Interact 2009c;9(4):225-35.

Belavý DL, Ng JK, Wilson SJ, Armbrecht G, Stegeman DF, Rittweger J, et al. Influence of prolonged bed-rest on spectral and temporal electromyographic motor control characteristics of the superficial lumbo-pelvic musculature. J Electromyogr Kinesiol 2010;20(1):170-9.

Belavý DL, Armbrecht G, Richardson CA, Felsenberg D, Hides JA. Muscle atrophy and changes in spinal morphology: is the lumbar spine vulnerable after prolonged bed-rest? Spine 2011;36(2):137-45.

Bernardi M, Solomonow M, Nguyen G, Smith A, Baratta R. Motor unit recruitment strategy changes with skill acquisition. Eur J Appl Physiol Occup Physiol 1996;74(1-2):52-9.

Blanc S, Normand S, Ritz P, Pachiaudi C, Vico L, Gharib C, et al. Energy and water metabolism, body composition, and hormonal changes induced by 42 days of enforced inactivity and simulated weightlessness. J Clin Endocrinol Metab 1998;83(12):4289-97.

Blottner D, Salanova M, Puttmann B, Schiffl G, Felsenberg D, Buehring B, et al Human skeletal muscle structure and function preserved by vibration muscle exercise following 55 days of bed rest. Eur J Appl Physiol 2006;97(3):261-71.

Booth FW, Gollnick PD. Effects of disuse on the structure and function of skeletalmuscle. Med Sci Sports Exerc 1983;15(5):415-20.

Carson RG, Riek S. Changes in muscle recruitment patterns during skill acquisition. Exp Brain Res 2001;138(1):71-87.

de Foa JL, Forrest W, Biedermann HJ. Muscle-fiber direction of longissimus, iliocostalis and multifidus - landmark-derived reference lines. J Anat 1989;163(April):243-7.

de Luca CJ. The use of surface electromyography in biomechanics. J Appl Biomech 1997;13(2):135-63.

Fontana TL, Richardson CA, Stanton WR. The effect of weight-bearing exercise with low frequency, whole body vibration on lumbosacral proprioception: a pilot study on normal subjects. Aust J Physiother 2005;51(4):259-63.

Gabriel DA, Boucher JP. Practice effects on the timing and magnitude of antagonist activity during ballistic elbow flexion to a target. Res Q Exerc Sport 1998;69(1):30-7.

Gentili R, Papaxanthis C, Pozzo T. Improvement and generalization of arm motor performance through motor imagery practice. Neuroscience 2006;137(3): 761-72.

Gribble PL, Mullin LI, Cothros N, Mattar A. Role of cocontraction in arm movement accuracy. J Neurophysiol 2003;89(5):2396-405.

Grimaldi A. MRI investigations of the muscles involved in lateral stability of the hip. PhD Thesis, Brisbane: School of Health \& Rehabilitation Sciences, The University of Queensland; 2008.

Hemingway MA, Biedermann $\mathrm{HJ}$, Inglis J. Electromyographic recordings of paraspinal muscles: variations related to subcutaneous tissue thickness. Biofeedback Self-regulation 1995;20(1):39-49.

Hides JA, Belavý DL, Stanton W, Wilson SJ, Rittweger J, Felsenberg D, et al. MRI assessment of trunk muscles during prolonged bed rest. Spine 2007;32(15): 1687-92.

Hodges PW, Gandevia SC. Activation of the human diaphragm during a repetitive postural task. J Physiol 2000;522(Pt 1):165-75

Ludwig DA. EMG changes during acquisition of a motor skill. Am J Phys Med 1982;61(5):229-43.

Lyons K, Perry J, Gronley JK, Barnes L, Antonelli D. Timing and relative intensity of hip extensor and abductor muscle action during level and stair ambulation. An EMG study. Phys Ther 1983;63(10):1597-605. 
Mulder ER, Stegeman DF, Gerrits KH, Paalman MI, Rittweger J, Felsenberg D, et al. Strength, size and activation of knee extensors followed during 8 weeks of horizontal bed rest and the influence of a countermeasure. Eur J Appl Physiol 2006;97(6):706-15.

$\mathrm{Ng}$ JK-F, Kippers V, Richardson CA. Muscle fibre orientation of abdominal muscles and suggested surface EMG electrode positions. Electromyogr Clin Neurophysiol 1998;38(1):51-8.

Ng JK-F, Parnianpour M, Richardson CA, Kippers V. Functional roles of abdominal and back muscles during isometric axial rotation of the trunk. J Orthop Res 2001;19(3):463-71.

Ng JK-F, Richardson CA, Parnianpour M, Kippers V. EMG activity of trunk muscles and torque output during isometric axial rotation exertion: a comparison between back pain patients and matched controls. J Orthop Res 2002;20(1): 112-21.

Nicogossian AE, Dietlein LF. Microgravity simulation and analogues. In: Nicogossian AE, editor. Space physiology and medicine. Philadelphia: Lea \& Febiger; 1982. p. 240-8.

Nordander C, Willner J, Hansson GA, Larsson B, Unge J, Granquist L, et al. Influence of the subcutaneous fat layer, as measured by ultrasound, skinfold calipers and BMI, on the EMG amplitude. Eur J Appl Physiol 2003;89(6):514-9.

Pinheiro JC, Bates DM. Mixed-effects models in $S$ and S-PLUS. 1st ed. Berlin: Springer; 2000.

Richardson C, Bullock MI. Changes in muscle-activity during fast, alternating flexion extension movements of the knee. Scand J Rehabil Med 1986;18(2):51-8

Richardson CA. Investigations into the optimal approach to exercise for the knee musculature. PhD Thesis. Brisbane, Australia: Department of Physiotherapy, The University of Queensland; 1987.

Rittweger J, Belavy DL, Hunek P, Gast U, Boerst H, Feilcke B, et al. Highly demanding resistive vibration exercise program is tolerated during 56 days of strict bedrest. Int J Sports Med 2006;27(7):553-9.

Rittweger J, Beller G, Armbrecht G, Mulder E, Buehring B, Gast U, et al. Prevention of bone loss during 56 days of strict bed rest by side-alternating resistive vibration exercise. Bone 2010;46(1):137-47.

Vink P, Daanen HAM, Verbout AJ. Specificity of surface-EMG on the intrinsic lumbar back muscles. Hum Mov Sci 1989;8(1):67-78.

Vinther A, Kanstrup IL, Christiansen E, Alkjaer T, Larsson B, Magnusson SP, et al. Exercise-induced rib stress fractures: influence of reduced bone mineral density. Scand J Med Sci Sports 2005;15(2):95-9.

Winter DA. Biomechanics and motor control of human movement. 3rd ed. New Jersey: John Wiley \& Sons; 2004

Winter DA, Yack HJ. EMG profiles during normal human walking: stride-to-stride and inter-subject variability. Electroencephalogr Clin Neurophysiol 1987;67(5): 402-11.

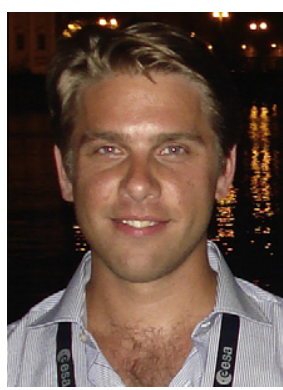

Daniel L. Belavý is currently a post-doctoral research associate at the Charite University Medicine, Berlin. His interests are in musculoskeletal changes in weightlessness, countermeasures against these changes and he has also published in the fields of signal/ data analysis. He enjoys his work but also enjoys taking it easy.

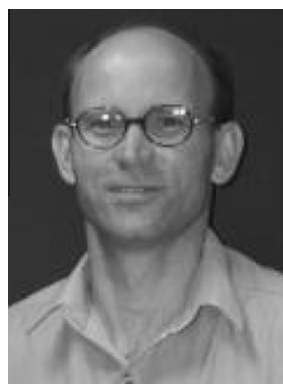

Stephen J. Wilson is a medically qualified engineer and medical doctor, is currently associate professor in the School of Information Technology and Electrical Engineering at the University of Queensland. Instrumentation and imaging for musculoskeletal measures is one theme of his research. He also participates in sleep and respiratory medicine based research projects and pursues interests in nonlinear biological signal analysis, biomedical instrumentation generally and engineering teaching at undergraduate level.

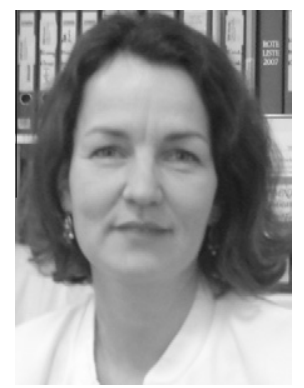

Gabriele Armbrecht received her M.D. and Ph.D. at the Medical School, Free University, Berlin, Germany and is currently in her last year for board certification in radiology. Since 2004 she is vice-leader of the Centre for Muscle and Bone Research at the Charité University Medicine, Berlin. Her special interests are in radiological diagnostic techniques for detection of muscle and bone changes.

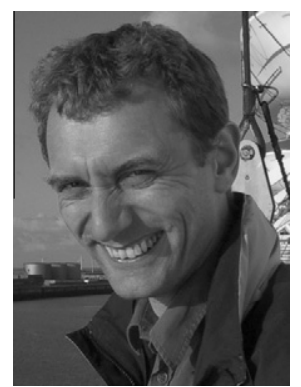

Jörn Rittweger received his M.D. from the LudwigMaximilians University in Munich, Germany, and his Ph.D. in physiology from Charité University Medicine in Berlin. He is currently working as a Head of the division "Space Physiology" in the Institute of Aerospace Medicine at the German Aerospace Center (DLR) in Cologne, Germany.

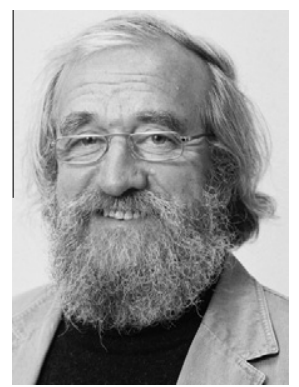

Dieter Felsenberg is leader of the Centre for Muscle and Bone Research and Professor at the Charite University Medicine, Berlin. His research focuses on osteoporosis, bone and muscle metabolism, bone biomechanics, diagnostics of bone metastases, sports medicine, rheumatoid arthritis, micro-CT technology and muscle and bone metabolism in weightlessness. He is a member of a number of European and American radiological and osteoporosis societies, is vicepresident of the German Academy of Osteology and Rheumatology (DAdorW) and president of the German Society of Muscle and Bone Research.

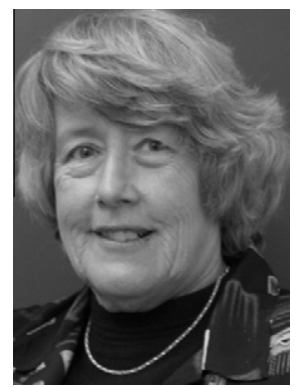

Carolyn A. Richardson focuses on into the most effective and efficient exercise treatment (and countermeasures) for musculoskeletal injuries, especially low back pain, which are linked to inadequate stabilisation and support of the joints. Current research has developed a new focus relating to the function of the human antigravity muscle system, which is severely affected when gravity is minimized (including in microgravity). These changes provide new information on the possible aetiology of low back pain, as well as other conditions such as osteoarthritis of the weightbearing joints and osteoporosis. This line of research has led to extensive research collaboration and consultancies with the European Space Agency (ESA), including scientific consultant to the Toulouse Bedrest study, member of the ESA Topical Team for Low Back Pain, Senior Collaborator and on the Board of the ESA project "Vibration Exercise in Space", a Bedrest study, undertaken at the Free University of Berlin February 2003 to May 2005. 
Table 1: Baseline (BR1) movement accuracy

\begin{tabular}{|c|c|c|c|c|c|c|}
\hline \multirow{2}{*}{ Movement-speed } & \multicolumn{6}{|c|}{ Movement accuracy variables } \\
\hline & $M S E_{0^{\circ}}$ & $M S E_{45^{\circ}}$ & $M S E_{\text {speed }}$ & All-MSE $0^{\circ}$ & All-MSE $_{45^{\circ}}$ & All-MSE $E_{\text {speed }}$ \\
\hline \multicolumn{7}{|c|}{ Inactive control group (CTRL) } \\
\hline $50 \mathrm{cyc} / \mathrm{min}$ & $0.81(0.41)$ & $1.78(0.23)$ & $6.39(0.83)$ & $0.77(0.23)$ & $2.06(0.67)$ & $10.81(2.43)$ \\
\hline $75 \mathrm{cyc} / \mathrm{min}$ & $1.21(0.43)$ & $1.52(0.22)$ & $6.75(0.71)$ & $1.32(0.27)$ & $2.82(0.69)$ & $21.88(5.17) *$ \\
\hline $100 \mathrm{cyc} / \mathrm{min}$ & $1.94(0.43)^{*}$ & $2.37(0.25)$ & $6.38(0.69)$ & $2.13(0.34) \ddagger$ & $3.66(0.77)$ & $26.46(6.20)^{*}$ \\
\hline $125 \mathrm{cyc} / \mathrm{min}$ & $3.80(0.50)+$ & $3.20(0.31) \ddagger$ & $7.28(0.85)$ & $4.38(0.47) \ddagger$ & $6.06(0.90) \div$ & $83.47(18.84) \ddagger$ \\
\hline \multicolumn{7}{|c|}{ Resistive vibration exercise group (RVE) } \\
\hline $50 \mathrm{cyc} / \mathrm{min}$ & $1.54(0.31)$ & $2.16(0.30)$ & $4.03(0.58)$ & $1.59(0.34)$ & $2.91(0.51)$ & $6.86(1.49)$ \\
\hline $75 \mathrm{cyc} / \mathrm{min}$ & $1.16(0.31)$ & $2.77(0.32)$ & $6.82(0.72)$ & $1.44(0.34)$ & $4.33(0.73)$ & $13.57(2.77)^{*}$ \\
\hline $100 \mathrm{cyc} / \mathrm{min}$ & $2.54(0.37)^{*}$ & $3.39(0.32)^{*}$ & $6.16(0.65)$ & $2.95(0.48)^{*}$ & $5.31(0.54) \dagger$ & $20.57(3.56) \dagger$ \\
\hline $125 \mathrm{cyc} / \mathrm{min}$ & $2.30(0.44)$ & $3.98(0.36) \dagger$ & $7.40(1.11)$ & $3.76(0.67) \dagger$ & $7.69(0.96) \dagger$ & $93.93(18.23) \dagger$ \\
\hline
\end{tabular}

Values are mean(SEM). $M S E_{0^{\circ}}$ and $M S E_{45^{\circ}}$ variables are in degrees $M S E_{\text {speed }}$ variables are in cycles per minute. ${ }^{*}: p<0.05 ; \dagger: p<0.01 ; \ddagger: p<0.001$ and indicates significance of difference to value at $50 \mathrm{cyc} / \mathrm{min} . M S E_{45^{\circ}}$ was higher at baseline in the RVE group $(p<0.001)$, otherwise there we no differences between the groups at baseline $(p \geq 0.053) . M S E_{0^{\circ}}$ and $M S E_{45^{\circ}}$ and $M S E_{\text {speed }}$ are meansquared error around their respective extension position, flexion position and speed target targets within the sections of the goniometer trace which were defined as "acceptable" for further analysis of EMG data (see Methods). All-MSE $0^{\circ}$ and All-MSE $45^{\circ}$ and All-MSE $E_{\text {speed }}$ represent mean-squared error of entire goniometer trace around their respective targets from each movement trial. All-MSE variables were used to track changes in movement performance over the course of the study (see supplementary online material Figure 2), whereas the MSE variables were generated solely to correlate with the corresponding EMG variables at baseline (see supplementary online material Table $3)$. 
Table 2: Baseline (BR1) electromyographic parameters

\begin{tabular}{ccccc}
\hline \hline \multirow{2}{*}{$\begin{array}{c}\text { Movement-speed } \\
\text { (cyc/min) }\end{array}$} & \multicolumn{2}{c}{ RMS (mV) } & \multicolumn{2}{c}{ PHZ (degrees) } \\
\cline { 2 - 4 } & CTRL & RVE & CTRL & RVE \\
\hline
\end{tabular}

\section{External Oblique}

$50 \mathrm{cyc} / \mathrm{min}$

$75 \mathrm{cyc} / \mathrm{min}$ $100 \mathrm{cyc} / \mathrm{min}$

$125 \mathrm{cyc} / \mathrm{min}$

$50 \mathrm{cyc} / \mathrm{min}$ $75 \mathrm{cyc} / \mathrm{min}$ $100 \mathrm{cyc} / \mathrm{min}$ $125 \mathrm{cyc} / \mathrm{min}$

$50 \mathrm{cyc} / \mathrm{min}$ $75 \mathrm{cyc} / \mathrm{min}$ $100 \mathrm{cyc} / \mathrm{min}$ $125 \mathrm{cyc} / \mathrm{min}$

$50 \mathrm{cyc} / \mathrm{min}$ $75 \mathrm{cyc} / \mathrm{min}$ $100 \mathrm{cyc} / \mathrm{min}$ $125 \mathrm{cyc} / \mathrm{min}$
$5.40(1.16) \quad 4.76(0.60) \quad 164.24(121.14) 103.41(119.17)$ $\begin{array}{llll}6.80(1.47) * & 5.77(0.74) & -91.22(88.76) & -27.60(30.99)\end{array}$ $11.55(2.51) \div 8.37(1.08) \dagger-79.77(78.28)-77.51(70.07)$ $19.23(4.29) \$ 15.21(2.37) \$-75.85(74.76)-47.46(47.05)$

Inferior Gluteus Maximus

$1.43(0.21) \quad 1.22(0.08) 150.63(148.41) \quad$ \#DIV/0! $1.98(0.29) \quad 1.54(0.11) * 131.21(133.05) 92.96(94.17)$ $3.46(0.51) \$ 2.29(0.16) \$ 128.44(130.68) 117.35(116.89)$ $6.52(1.04) \$ 4.14(0.36) \$ 138.51(139.33) 133.23(131.99)$

\section{Internal Oblique}

7.77(1.17) 3.41(0.43) $-177.45(93.61) \quad 80.77(87.61)$ $12.88(1.94) * 5.53(0.72) \dagger 126.47(125.25)-43.13(76.64)$ $23.36(3.52)+9.48(1.23) \div-97.68(86.19)-20.35(58.53)$ $34.41(5.60) \$ 14.76(2.22) \$-98.97(88.85)-61.45(70.41)$

\section{Lumbar Erector Spinae}

$2.99(1.07) \quad 1.88(0.30) \quad 171.76(156.74) 144.37(143.44)$ $6.76(2.41) \dagger 7.55(1.22) \div 118.40(122.17) 122.89(122.89)$ $13.89(4.96) \$ 20.78(3.23) \$ 122.11(124.98) 127.25(127.20)$ $21.48(7.88) \$ 33.09(5.86) \$ 133.37(134.71) 134.05(133.91)$ 
Thoracic Erector Spinae

$$
\begin{array}{ccccc}
50 \mathrm{cyc} / \mathrm{min} & 3.84(0.68) & 2.95(0.42) & -90.00(79.24) & -25.43(26.29) \\
75 \mathrm{cyc} / \mathrm{min} & 4.90(0.87) & 3.81(0.55) & -131.71(99.09) 104.86(112.74) \\
100 \mathrm{cyc} / \mathrm{min} & 7.41(1.32) \$ 8.79(1.29) \$ 166.21(130.95) 131.06(131.57) \\
125 \mathrm{cyc} / \mathrm{min} & 11.87(2.24) \$ 15.97(2.76) \$ 159.61(129.01) 131.76(132.52)
\end{array}
$$

Values are mean(SEM) root-mean-square (RMS) EMG amplitude and activation timing (phase-lead/lad, PHZ; or where EMG peaks occur relative to peaks in EMG signal). Activation time (PHZ) could not be measured for all muscles at all measurement speeds as sufficient amplitude modulation of the EMG signal must be present in order to detect a phase-lead/lag of the EMG trace relative to the goniometer trace ("-" indicates missing data). These PHZ data are from $100 \mathrm{~Hz}$ high-pass filter. Internal oblique RMS ( $p=0.007)$ was higher on average in the CTRL group at

5 baseline with the LES-RMS showing a different response with movement-speed in the two groups at baseline ( $\mathrm{p}=0.037)$. Otherwise there were no significant differences between the groups at baseline $(p \geq 0.074)$. 
Table 3: Correlations between movement accuracy and electromyographic parameters at baseline (BR1)

\begin{tabular}{cccccccc}
\hline \hline Parameter & $M S E_{0^{\circ}}$ & $M S E_{45^{\circ}}$ & $M S E_{\text {speed }}$ & RMS & CoCon & BTR & ratio \\
\cline { 2 - 8 }$M S E_{0^{\circ}}$ & & -0.01 & -0.15 & -0.09 & 0.16 & 0.01 & -0.08 \\
$M S E_{45^{\circ}}$ & 0.41 & & 0.33 & 0.1 & 0.01 & 0.13 & 0.08 \\
$M S E_{\text {speed }}$ & 0.11 & 0.27 & & -0.04 & -0.21 & 0.09 & 0.04 \\
RMS & 0.25 & 0.27 & 0.12 & & -0.02 & 0.37 & 0.21 \\
CoCon & -0.34 & -0.41 & -0.22 & -0.34 & & -0.27 & -0.1 \\
BTR & 0.2 & 0.28 & 0.12 & 0.52 & -0.39 & & 0.22 \\
ratio & 0.01 & 0.13 & 0.09 & 0.25 & -0.25 & 0.29 & \\
\hline
\end{tabular}

Values are Pearson's correlation co-efficient. Values in bottom left (grey background) triangle are correlations between parameters for all muscles and movement-speeds. Values in top right triangle are Pearson's partial correlation co-efficients controlling for movement-speed and muscle. Correlations are for descriptive purposes only and hence testing of statistical significance was not performed. Correlations with activation timing $(\mathrm{PHZ})$ variable could not be performed as this is a circular variable. Note that movement accuracy variables $\left(M S E_{0^{\circ}}\right.$,

$10 M S E_{45^{\circ}}, M S E_{\text {speed }}$ ) are not correlated with EMG variables once confounding factors (such as movement-speed) are controlled for. 
Table 4: Correlations between trunk fat mass and electromyographic variables at baseline (BR1)

\begin{tabular}{ccccc}
\hline \hline Muscle & RMS & CoCon & BTR & ratio \\
\hline & & & & \\
External Oblique & -0.14 & - & -0.27 & 0.19 \\
Internal Oblique & -0.33 & - & 0.02 & -0.07 \\
Inferior Gluteus Maximus & -0.25 & - & -0.09 & -0.16 \\
Lumbar Erector Spinae & -0.08 & - & -0.16 & -0.11 \\
Thoracic Erector Spinae & -0.06 & - & 0.15 & 0.32 \\
All muscles pooled & -0.28 & 0.05 & -0.17 & -0.04
\end{tabular}

Values are Pearson's correlation co-efficient. EMG variables were averaged across speeds within each subject and muscle prior to comparison to trunk fat mass. For "all muscles pooled", values were averaged within each subject across all muscles. Correlations are for descriptive purposes only and hence testing of statistical significance was not performed. Correlations with activation timing ( $P H Z$ ) variable could not be performed as this is a circular variable. Note that root-mean-square (RMS) EMG amplitude is consistently negatively correlated with fat mass (i.e. increases in fat mass are associated with lower activity levels). EMG amplitude modulation (BTR) and activity ratios (ratio) do not however show this consistent pattern and the extent of lumbo-pelvic extensor and flexor co-contraction (CoCon) appears unrelated to trunk fat mass. 
Table 5: Activation timing (phase-lead/lag; PHZ) over the course of the study

\begin{tabular}{|c|c|c|c|c|c|c|c|c|c|c|c|c|c|}
\hline \multirow{2}{*}{ Group } & \multicolumn{13}{|c|}{ Study-date } \\
\hline & BR1 & BR4 & BR13 & BR27 & BR41 & BR53 & $\mathrm{R}+3$ & $\mathrm{R}+7$ & $\mathrm{R}+14$ & $\mathrm{R}+28$ & $\mathrm{R}+90$ & $\mathrm{R}+180$ & $\mathrm{R}+3$ \\
\hline & \multicolumn{13}{|c|}{$100 \mathrm{~Hz}$ high pass filter prior to futher processing } \\
\hline & \multicolumn{13}{|c|}{ External Oblique } \\
\hline CTRL & $-85(-35,-105)$ & $-86(-53,23)$ & $-81(-46,21)$ & $-82(-52,22)$ & $-91(-17,47)$ & $-91(-16,45)$ & $-87(-44,20)$ & $-109(-17,49)$ & $-104(-17,48)$ & $-120(-18,52)$ & $-73(-50,21)$ & $-136(-17,53)$ & $-69(-6$ \\
\hline \multirow[t]{2}{*}{ RVE } & $-49(0,-88)$ & $-83(121,48)^{*}$ & $-106(1,293)^{*}$ & $-64(-46,41)$ & $-97(0,171)$ & $-125(1,-13)$ & $178(44,307)^{*}$ & $-169(0,182)^{*}$ & $152(42,311)^{*}$ & $-114(0,4)$ & $-104(0,182)^{*}$ & $-96(-1,108)$ & $-116(-1$ \\
\hline & & \multicolumn{12}{|c|}{ Inferior Gluteus Maximus } \\
\hline CTRL & $131(151,112)$ & $127(-18,17)$ & $133(-18,17)$ & $137(-17,16)$ & $135(-18,17)$ & $134(-16,15)$ & $139(-15,14)$ & $139(-17,16)$ & $130(-15,14)$ & $132(-16,15)$ & $128(-17,16)$ & $140(-19,17)$ & 124( \\
\hline \multirow{2}{*}{\multicolumn{14}{|c|}{ Internal Oblique }} \\
\hline & & & & & & & & & & & & & \\
\hline CTRL & $-97(-62,153)$ & $-180(-36,102)$ & $-118(-36,98)$ & $-120(-35,97)$ & $-143(-36,98)$ & $-174(-35,95)$ & $-175(-35,94)$ & $-173(-35,97)$ & $-140(-36,96)$ & $-161(-35,95)$ & $168(25,244)^{*}$ & $95(26,243)^{*}$ & $151(2$ \\
\hline RVE & $67(110,-16)$ & $174(-130,14)$ & $131(-121,14)$ & $122(-125,13)$ & $78(-42,68)$ & $108(-122,14)$ & $77(-42,68)$ & $89(-43,70)$ & $116(-125,14)$ & $98(-120,13)$ & $93(-123,14)$ & $127(-126,14)$ & $155(-$ \\
\hline \multicolumn{14}{|c|}{ Lumbar Erector Spinae } \\
\hline CTRL & $133(152,116)$ & $140(-15,14)$ & $150(-14,12)$ & $144(-14,13)$ & $149(-13,12)$ & $147(-13,12)$ & $141(-13,12)$ & $142(-14,12)$ & $138(-13,12)$ & $137(-13,12)$ & $131(-14,12)$ & $130(-15,13)$ & $125(-$ \\
\hline RVE & $133(151,118)$ & $138(-14,13)$ & $142(-13,12)$ & $145(-12,11)$ & $144(-12,11)$ & $143(-12,11)$ & $140(-12,11)$ & $137(-12,11)$ & $131(-12,11)$ & $133(-12,11)$ & $134(-12,11)$ & $131(-13,12)$ & 135( \\
\hline \multicolumn{14}{|c|}{ Thoracic Erector Spinae } \\
\hline CTRL & $163(-159,112)$ & $141(-39,59)$ & $147(-34,51)$ & $161(-34,52)$ & $149(-35,51)$ & $176(-33,49)$ & $171(-34,50)$ & $172(-34,50)$ & $148(-34,50)$ & $165(-34,50)$ & $147(-35,52)$ & $122(-36,53)$ & 150( \\
\hline RVE & $128(159,105)$ & $133(-34,27)$ & $133(-28,23)$ & $139(-29,23)$ & $144(-29,23)$ & $139(-27,22)$ & $142(-29,23)$ & $131(-29,23)$ & $136(-28,22)$ & $135(-28,22)$ & $119(-28,23)$ & $125(-29,24)$ & $138(-4$ \\
\hline \multicolumn{14}{|c|}{$20 \mathrm{~Hz}$ high pass filter prior to futher processing } \\
\hline CTRL & $-46(-14,-76)$ & $-56(-43,40)$ & $-50(-31,30)$ & $-45(-32,32)$ & $-61(-32,33)$ & $-56(-30,30)$ & $-51(-30,30)$ & $-53(-30,30)$ & $-35(-30,29)$ & $-55(-30,30)$ & $-57(-30,29)$ & $-66(-31,31)$ & -62( \\
\hline RVE & $-52(-8,-87)$ & $-26(-49,41)$ & $-56(-43,37)$ & $-41(-44,36)$ & $-66(-44,38)$ & $-73(-43,36)$ & $-75(-43,36)$ & $-33(-43,37)$ & $-58(-42,36)$ & $-62(-41,34)$ & $-48(-44,37)$ & $-59(-42,35)$ & -40( \\
\hline \multicolumn{14}{|c|}{ Inferior Gluteus Maximus } \\
\hline CTRL & $115(138,94)$ & $111(-23,20)$ & $123(-21,19)$ & $134(-21,19)$ & $133(-24,19)$ & $120(-21,19)$ & $120(-21,19)$ & $129(-23,20)$ & $127(-23,20)$ & $131(-24,21)$ & $122(-23,21)$ & $115(-27,23)$ & $126(-$ \\
\hline RVE & $119(140,98)$ & $115(-27,23)$ & $113(-19,19)$ & $113(-20,19)$ & $111(-20,19)$ & $117(-19,18)$ & $130(-20,18)$ & $116(-19,18)$ & $116(-18,18)$ & $110(-18,17)$ & $117(-19,18)$ & $110(-18,17)$ & $96(-$ \\
\hline \multicolumn{14}{|c|}{ Internal Oblique } \\
\hline CTRL & $172(-135,58)$ & $-125(-198,-27)^{*}$ & $51(29,219)^{*}$ & $-93(-130,39)$ & $-86(57,-18)$ & $-58(58,38)^{*}$ & $-70(57,14)^{*}$ & $-122(-186,-17)^{*}$ & $-59(58,35)^{*}$ & $-84(58,-15)$ & $-165(-273,-103) *$ & $59(30,220)^{*}$ & $159(-$ \\
\hline RVE & $46(87,-4)$ & $154(-22,2)$ & $47(-39,37)$ & $58(-40,39)$ & $61(-38,36)$ & $77(-38,36)$ & $62(-37,35)$ & $65(-38,36)$ & $91(-25,2)$ & $53(-43,41)$ & $96(-24,2)$ & $79(-42,39)$ & 88( \\
\hline \multicolumn{14}{|c|}{ Lumbar Erector Spinae } \\
\hline CTRL & $126(145,110)$ & $138(-17,15)$ & $145(-16,15)$ & $145(-15,14)$ & $149(-15,13)$ & $151(-14,13)$ & $144(-14,13)$ & $143(-15,14)$ & $137(-14,13)$ & $136(-15,13)$ & $133(-15,14)$ & $137(-17,15)$ & $131(-1$ \\
\hline RVE & $135(151,120)$ & $143(-14,14)$ & $142(-14,14)$ & $147(-13,12)$ & $142(-13,13)$ & $150(-12,12)$ & $143(-12,12)$ & $140(-13,13)$ & $136(-12,12)$ & $135(-12,12)$ & $132(-13,13)$ & $137(-13,13)$ & $125(-1$ \\
\hline \multicolumn{14}{|c|}{ Thoracic Erector Spinae } \\
\hline CTRL & $-161(-94,163)$ & $160(-15,118)$ & $162(-14,107)$ & $162(-14,101)$ & $168(-15,102)$ & $-174(-66,35)$ & $179(-14,99)$ & $167(-14,102)$ & $154(-14,98)$ & $161(-14,103)$ & $168(-14,103)$ & $110(-14,105)$ & $149(-$ \\
\hline RVE & $148(174,117)$ & $150(-28,35)$ & $147(-26,33)$ & $157(-28,33)$ & $145(-26,32)$ & $156(-24,29)$ & $158(-24,30)$ & $141(-24,30)$ & $135(-24,29)$ & $140(-23,29)$ & $131(-25,30)$ & $142(-25,31)$ & 126( \\
\hline
\end{tabular}

Values are mean phase-lead/lad on each study-date in degrees. Values in brackets are $95 \%$ confidence interval of mean change compared to baseline: if the confidence interval does not cross zero, then the difference to baseline is "significant" at $p<0.05$. BR=bed-rest, R+=recovery. CTRL: inactive control group; RVE: resistive vibration exercise group. Two versions of the data have been presented: one using a 100Hz high-pass filter prior to further signal processing and 5 another using a $20 \mathrm{~Hz}$ high-pass filter (the latter used in prior work; Belavý et al, 2010). The $100 \mathrm{~Hz}$ high-pass filter is more effective in removing cardiac signals from the EMG trace which were often present in the external oblique, internal oblique and thoracic erector spinae signals. 
Figure 1: Number of datasets available for analysis

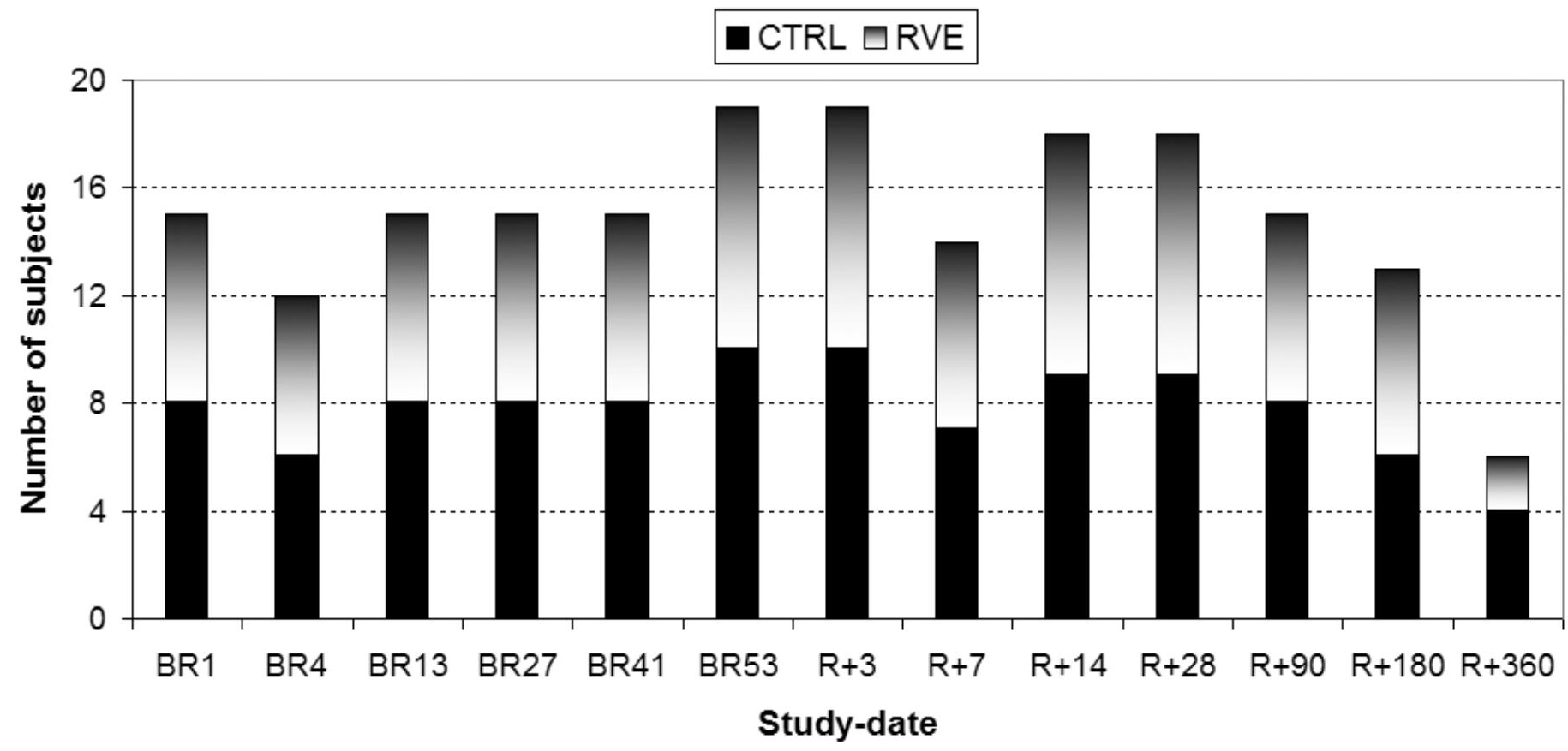

5 Due to subject absence and or technical issues, datasets were not available from all subjects on all measurement dates. CTRL: inactive control group; RVE: resistive vibration exercise group. 
Figure 2: Improvements in movement performance over the course of the study.
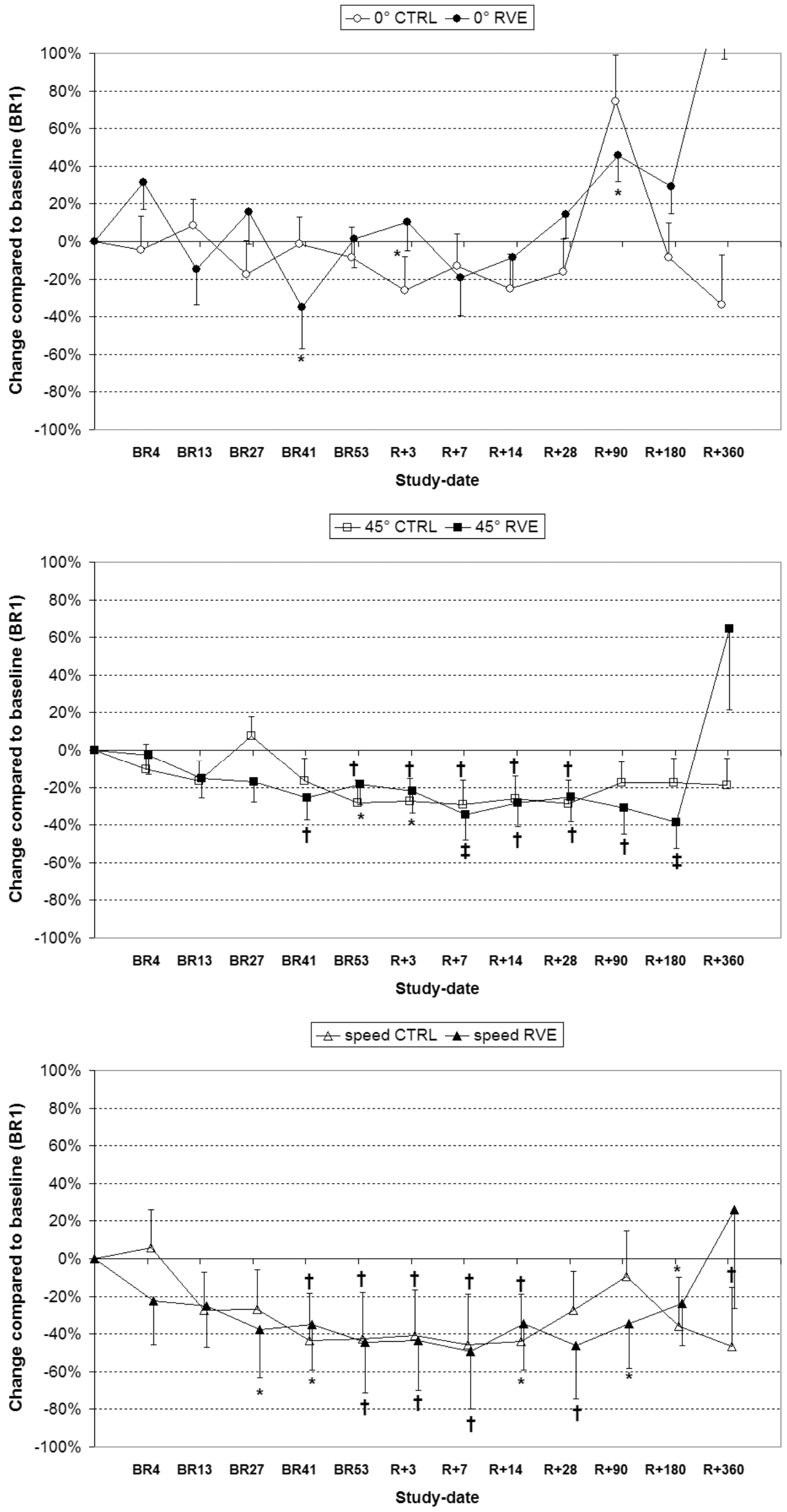
Values are mean(SEM) percentage difference to baseline (BR1) values. Data refer to mean-squared error (MSE) around targets from entire goniometer trace for extension (All-MSE $0_{0^{\circ}}$; top), flexion (All$M S E_{45^{\circ}}$; middle) and movement-speed (All-MSE $E_{\text {speed }}$; bottom). RVE-group R+360 All-MSE $0^{\circ}$ value was $141.3(44.3) \%$. See Table 1 for baseline (BR1) values. *: $p<0.05 ; \dagger: p<0.01 ; \ddagger: p<0.001$ and indicate significance of difference to baseline. $\mathrm{BR}=$ bed-rest, $\mathrm{R}+=$ recovery. $\mathrm{CTRL}$ : inactive control group; RVE: resistive vibration exercise group. With the exception of All-MSE $0_{0^{\circ}}(p=0.005$; see text), movement-speed was unrelated to the differences between groups over the course of the study. Neither All-MSE 45 $^{\circ}(p=0.09)$ nor All-MSE speed $(p=0.51)$ showed any between group differences. 
Figure 3: Changes in root-mean-square (RMS) amplitude in the lumbo-pelvic musculature

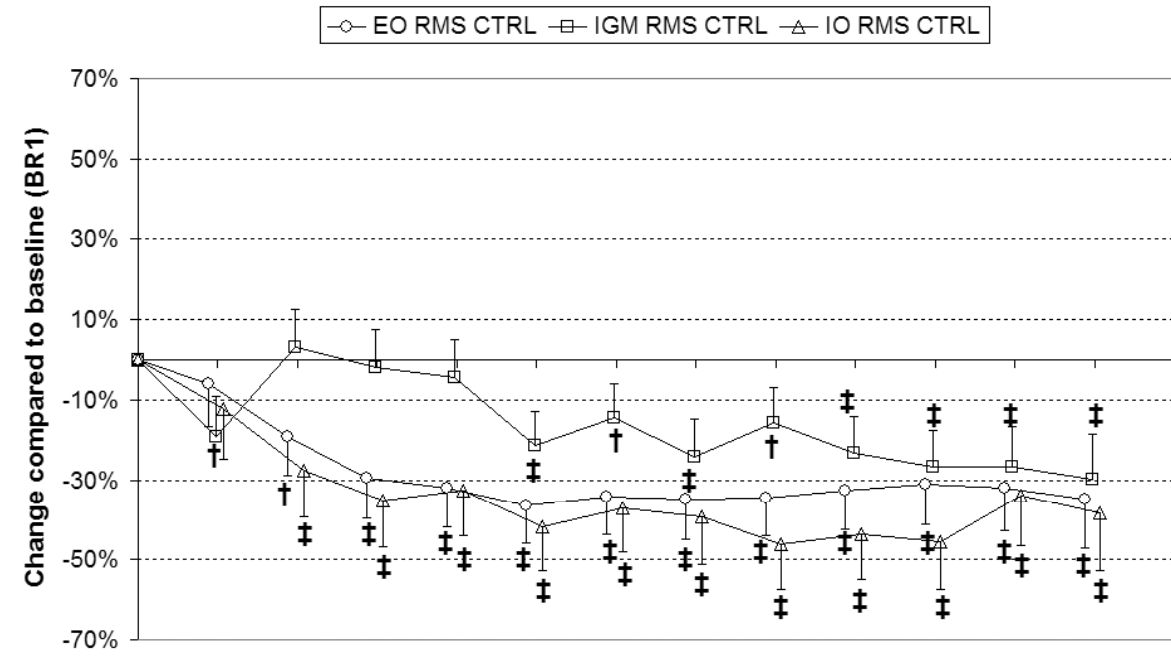

$\begin{array}{llllllllllll}\text { BR4 } & \text { BR13 } & \text { BR27 } & \text { BR41 } & \text { BR53 } & R+3 & R+7 & R+14 & R+28 & R+90 & R+180 & R+360\end{array}$ Study-date

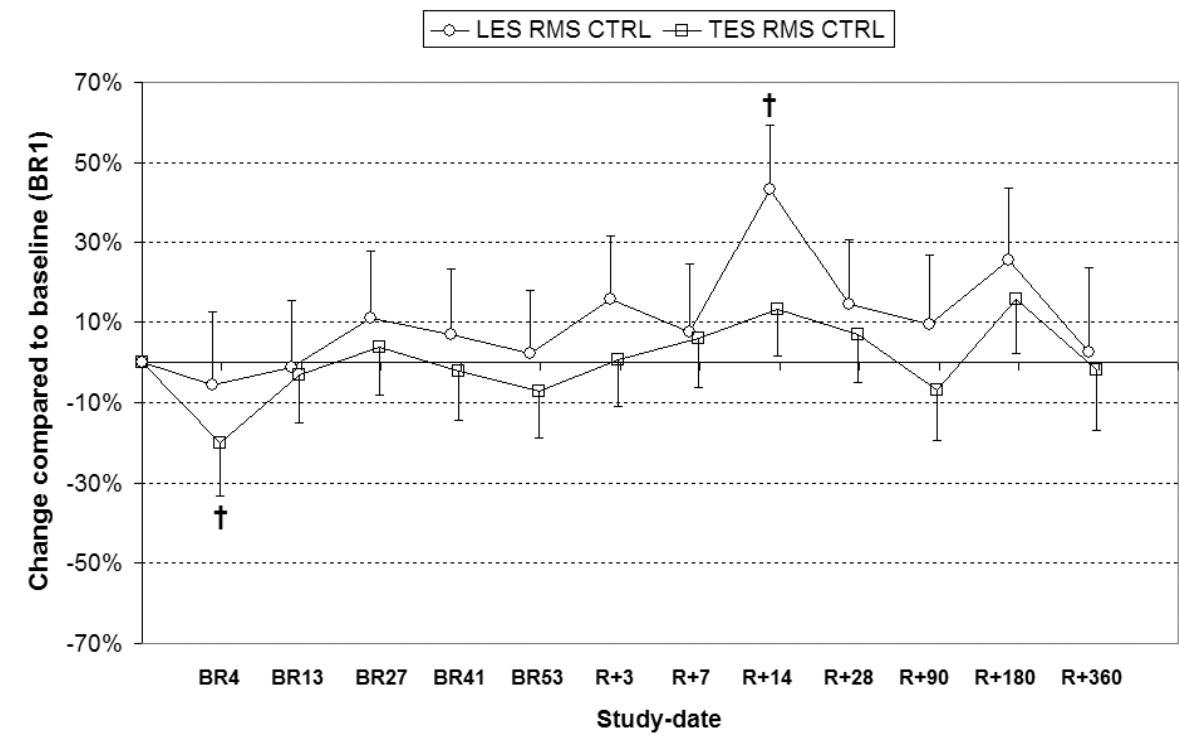

$\rightarrow-$ EO RMS RVE -- IGM RMS RVE $₫$ IO RMS RVE

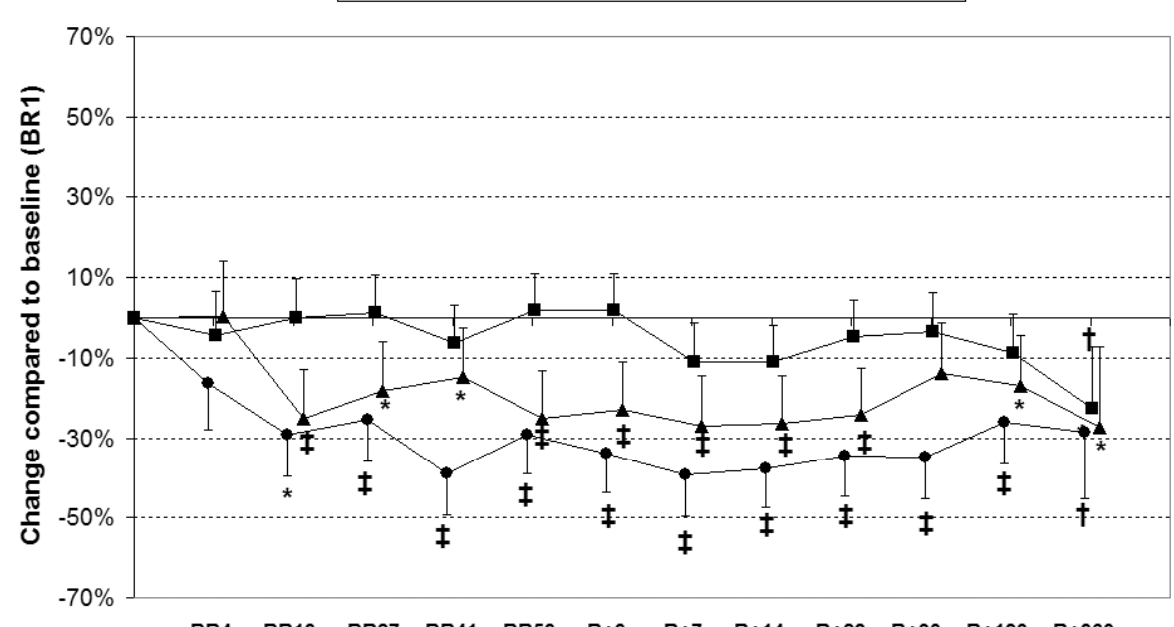

$\begin{array}{llllllllllll}\text { BR4 } & \text { BR13 } & \text { BR27 } & \text { BR41 } & \text { BR53 } & R+3 & R+7 & R+14 & R+28 & R+90 & R+180 & R+360\end{array}$ Study-date

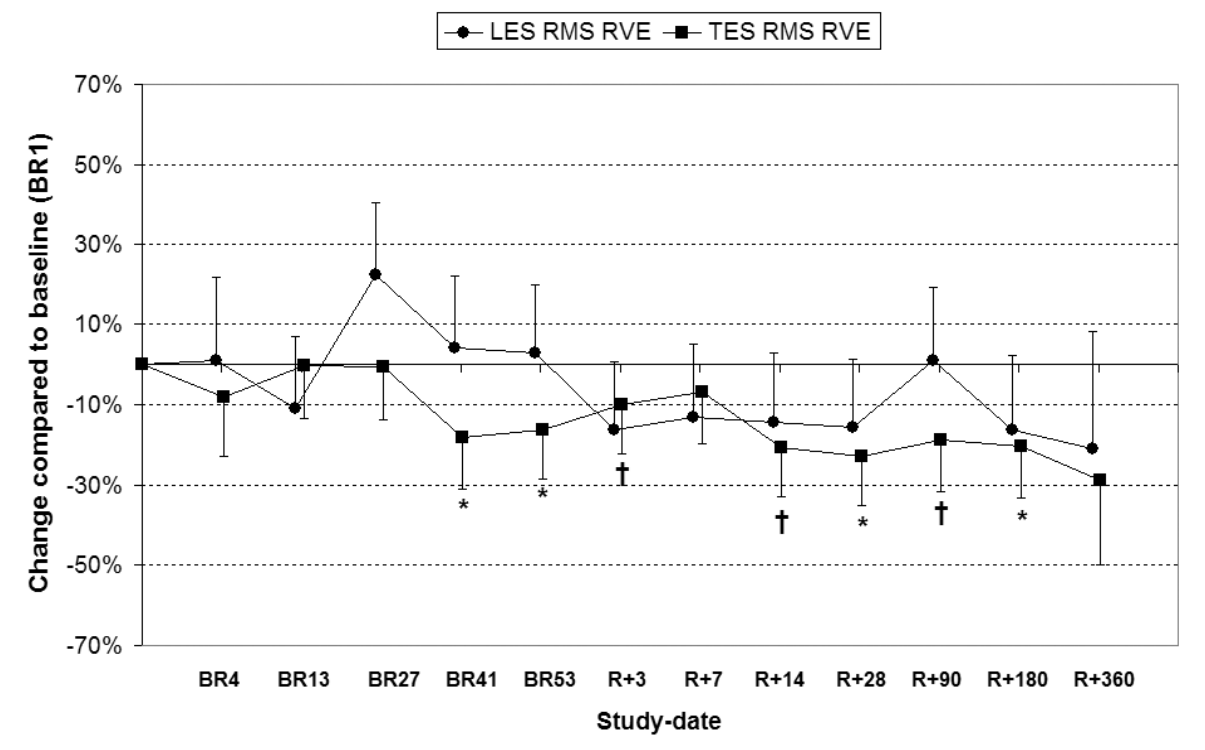


Values are mean(SEM) percentage difference to baseline (BR1) values. $*: p<0.05 ; \dagger: p<0.01 ; \ddagger: p<0.001$ and indicate significance of difference to baseline. BR=bed-rest, R+=recovery. CTRL: inactive control group (left); RVE: resistive vibration exercise group (right). EO: external oblique, IO: internal oblique, IGM: inferior gluteus maximus, TES: thoracic erector spinae, LES: lumbar erector spinae. Movement speed did not influence changes of each muscle over study-date $(p \geq 0.11)$, therefore results are averaged across movement speeds. ANOVA suggested a different response between groups over time for the different muscles (RMS: $p<0.001)$. Subsequent analyses for each muscle showed a significant group $\times$ study-date interaction for RMS for IO, IGM, LES and TES ( $\mathrm{p} \leq 0.018$; EO $p=0.26$ ). 